OPEN ACCESS

Edited by:

Peng Zhou,

Tsinghua University, China

Reviewed by:

Luka Crnic,

Hebrew University of Jerusalem, Israel

Rachel Dudley,

Central European University, Hungary

${ }^{*}$ Correspondence:

Yadav Gowda

ysg@mit.edu

Elise Newman

esnewman@mit.edu

Specialty section: This article was submitted to

Language Sciences,

a section of the journal

Frontiers in Communication

Received: 11 August 2020 Accepted: 23 November 2020

Published: 23 December 2020

Citation:

Gowda Y, Newman E, Rosenstein L and HackI M (2020) Scalar Inferences

in the Acquisition of Even.

Front. Commun. 5:593634.

doi: $10.3389 / f c o m m .2020 .593634$

\section{Scalar Inferences in the Acquisition of Even}

\author{
Yadav Gowda*, Elise Newman*, Leo Rosenstein and Martin Hackl \\ Department of Linguistics and Philosophy, Massachusetts Institute of Technology, Cambridge, MA, United States
}

Scalar inferences are ubiquitous in human reasoning. Correspondingly, language has many means of expressing and encoding them. One of these means is the focus particle even, which utilizes scalar inferences to signal the pragmatic status of asserted content as noteworthy. The vehicles that even employs to signal noteworthiness are scalar likelihood inferences. A peculiarity of these inferences is that they are presuppositional in nature (not-at-issue) and yet, they are responsive to the polarity of the sentence expressing the proposition whose likelihood is signaled. This property raises intricate questions about what learners might expect scalar operators of this sort to look like (initial hypothesis space) as well as what type of evidence and learning strategies they have access to as they figure out the specific properties of even in adult English. This paper presents a detailed study of this development, combining data from a series of comprehension experiments and corpus studies. We find that children are sensitive to the basic scalar nature of even much earlier than previous literature has claimed. We additionally find, however, that children sometimes exhibit non-adult-like responses to even sentences, which we argue provide insight into their developing grammar. On this view, the child grammar offers a larger option space for even than the adult grammar. Becoming adult-like, in turn, involves eliminating some of these options, namely those that are underutilized in production due to their limited conversational value.

Keywords: language acquisition, even, scalar inferences, presupposition, focus particles, additive particles, polarity

\section{INTRODUCTION}

This paper investigates the status of scalar inferences in child grammar through an acquisition study of the scalar focus particle even. English even provides a window through which to study the grammatical encoding of scalar inferences due to its sensitivity to polarity. It triggers a least-likely presupposition in positive sentences, and a most-likely presupposition in negative sentences as illustrated in (1) and (2), (Horn, 1969; Karttunen and Peters, 1979, a.o.).

(1) Jack had even invited AMY to the party. - Amy was the least-likely for Jack to invite to the party.

- There was at least one other person (out of a salient set) that Jack had invited to the party.

(2) Kim hadn't even invited SAM to the party. - Sam was the most-likely for Kim to invite to the party.

- There was nobody else (out of a salient set) that Kim had invited to the party.

The meaning conveyed in (1) and (2) consists of three components: the asserted content, which is just the ordinary meaning of the sentence without even (i.e., the meaning of prejacent), and two 
invited inferences-scalar and additive in nature-which do not have the status of at-issue content. Rather they exhibit signature properties of presuppositions (or conventional implicatures), which persist even when the presupposition trigger occurs in the scope of entailment-canceling operators. This can be readily seen when transforming these sentences into questions, (3) and (4), which still give rise to the same invited inferences ${ }^{1}$.

(3) Did Jack even invite AMY to the party?

- Amy was the least-likely for Jack to invite to the party.

- There was at least one other person (out of a salient set) that Jack had invited to the party.

(4) Did Kim not even invite SAM to the party?

- Sam was the most-likely for Kim to invite to the party. - There was nobody else (out of a salient set) that Kim has invited to the party.

Interestingly, even though the invited inferences triggered by even project in questions, their precise character is affected by the polarity of the host sentence. Concentrating on the scalar inferences, which are the focus of this paper, we observed in (1) and (2) that the presence or absence of negation corresponds to a "most- or least-likely" inference, respectively. The fact that these are different seems to suggest that the content upon which the scalar inference is based is visible to negation, though not in a way that would cancel the inference as is the case for asserted content.

This interaction between likelihood presupposition and polarity has generated much discussion in the theoretical literature. It also raises rather interesting questions about how learners acquire the full pattern of invited inferences triggered by even: What is the initial hypothesis space that learners need to navigate as they acquire even ${ }^{2}$ What is the evidence they have access to and make use of to transform their initial state of the grammar into the adult state, and what are the learning strategies that allow them to do that? We aim to address these questions via a series of comprehension and corpus studies (complemented by adult control experiments) that allow us to establish the developmental path children follow as they learn to track the different likelihood inferences triggered by even across different environments. Our findings portray a much richer and more intricate acquisition process than previous work has suggested, making it possible to identify the specific structure of the initial hypothesis space for even as well as the grammatical and pragmatic factors that enable and constrain the learning.

\footnotetext{
${ }^{1}$ There is a lively debate as to the precise content, origin and status of the scalar and additive inferences. Since much of this debate is orthogonal to our purpose here, we label them either as "invited inferences" or as presuppositions. Moreover, we largely abstract away from the additive inference. See among others (Horn, 1972, 1989; Karttunen and Peters, 1979; Rooth, 1985; Krifka, 1991; von Stechow, 1991; Rullmann, 1997, 2007; Herburger, 2000; Schwarz, 2005; Greenberg, 2016, 2018; Francis, 2018).

${ }^{2}$ Recent years have seen an impressive growth of cross-linguistic work on additive scalar particles like English even (Giannakidou, 2007; Crnič, 2009; Gast and van der Auwera, 2011; Greenberg, 2015, 2018, a.o.). A more complete framing of the initial hypothesis space for even would situate the current discussion within that typology. However, since the cross-linguistic inventory of such particles is rather rich and there is no consensus yet on the organizational principles spanning that typology, we will have to leave it to future work.
}

To foreshadow, our comprehension studies demonstrate that preschool-aged children show clear evidence of sensitivity to scalar inferences associated with even, contrary to what previous research has suggested. They also reveal a rather nuanced developmental trajectory traceable already in 4yos regarding the at times nonadult-like nature of the scalar inferences. Importantly, our comprehension tasks reveal that not all non-adult-like behavior should be given the same analysis. While certain responses appear to indicate true confusion about even, other responses appear systematic and informed by their developing grammar, despite being non-adult-like. This is most clearly evident from the fact that these responses are accompanied by reasoned justifications involving reference to scalar properties.

Our adult control studies present a similar finding. They, too, show a systematic error pattern during real time comprehension (albeit at a lower rate) which appears to be a function of the adultgrammar of even. We argue on the basis of their systematicity and their similarities that both the non-adult-like inferences generated by children and the corresponding errors in our adult studies reflect options made available by the basic architecture of the respective grammars of even. Hence, they should inform our theories of adult and child even, and put together, they should frame accounts of how even is acquired.

Our conclusions from the comprehension experiments are further enriched by our corpus studies on child and childdirected adult use of even, presented in section 5. We find that children who produce even do so essentially error-free, even at 3-4 years of age. We additionally find that the form of our stimuli in the comprehension studies instantiates a lowfrequency use pattern for even in both children and adults. The fact that children nevertheless show partial command of even when their comprehension is tested on these items suggests that children in our age range already have a quite robust grasp of the fundamental fact the even always triggers a scalar inference of some sort.

Most striking about the corpus data is, however, that children (and adults) do not exhibit the non-adult-like behavior that we find in the comprehension experiments. At first sight, this constitutes a rather surprising production-comprehension asymmetry: the child grammar appears adult-like in production but non-adult-like in comprehension. We propose to resolve this puzzle by assuming that it is the comprehension data that faithfully reflect the child grammar of even, which is non-adultlike in that it provides a larger space of options for even than the adult grammar. The fact that their production data is fully adult-like should, in turn, be seen as the result of child speakers choosing not to realize some of the grammatically licit options. We suggest that they underutilize some of these options because of their limited pragmatic utility. To explain why production is ahead of comprehension, we suggest that the pragmatic oddity of these options is more transparent from the perspective of the (child) speaker than from the perspective of the (child) listener since the speaker knows the intended message while the listener needs to infer it from the form of the utterance and the presumed conversational goals of the speaker. 


\section{EVEN'S SCALAR INFERENCES: THEORY AND ACQUISITION}

\subsection{Theoretical Background}

Our study's main focus is the interaction between even's scalar inferences and sentential polarity as it relates to acquisition. As argued in Karttunen and Peters (1979) the scalar inferences have the status of a presupposition/conventional implicature. This can be inferred, for instance, from the fact that, unlike at issue content, they survive embedding inside entailment canceling environments such as questions, (3-4). Interestingly, and somewhat unexpectedly the precise nature of the scalar inference is sensitive to the presence of negation (an entailment canceling operator in its own right). Recalling the examples in (1) and (2) we see that the scalar inference in (1) is substantially different from the one in (2).

(1) Jack had even invited AMY to the party. - Amy was the least-likely for Jack to invite to the party.

(2) Kim hadn't even invited SAM to the party.

- Sam was the most-likely for Kim to invite to the party.

In both (1) and (2), we detect an inference about the likelihood of somebody having been invited to a party. However, the inference in (2) is essentially the opposite of that in (1). While Amy is understood to be someone that was unlikely to be invited, Sam is understood to be someone who was very likely to be invited. Both (1) and (2) therefore convey something surprising, namely that someone was invited who wasn't expected to be invited, or that someone wasn't invited who was expected to be invited.

There are two families of approaches that attempt to capture this property of even. The first type of approach (Karttunen and Peters 1979, henceforth the scope theory) argues that even uniformly triggers a least-likely presupposition, but has a requirement that it outscope (clause-mate) negation ${ }^{3}$ resulting in a most-likely inference. We can schematize this approach as in (5)-(7). Even is assumed to be a clausal operator that combines with a propositional argument (the prejacent) and a set of alternative propositions (derived from the prejacent via focus semantics ${ }^{4}$ ). Its lexical semantics is that of a filter which passes on the meaning of its propositional argument $p$ unchanged but only if $p$ is the least likely member in $C,(6)$. For positive sentences this

${ }^{3}$ See Wilkinson (1996), Lahiri (1998), Guerzoni (2004), a.o. A complete description says that even needs to outscope all downward-entailing operators, not just negation. Consider example (i) where even is inside a conditional. An in situ treatment of even makes the wrong (and contradictory) prediction that Mary is less likely to notice one mistake than she is to notice multiple mistakes. In fact, we infer that she is most-likely to infer one mistake over many.

(i) If Mary noticed even one e $_{F}$ mistake of yours, it would be a problem. (Guerzoni, 2004)

- Mary noticing one mistake is most-likely compared to noticing multiple mistakes.

On the scope theory, the most-likely inference in (i) is explained if even moves outside the scope of the conditional.

${ }^{4}$ Since the fine mechanics of focus prosody and focus semantics are not central to our paper we abstract away from the details here and refer the reader to e.g., Rooth, 1996 etc. delivers a least-likely inference. For negated sentences, however, with even scoping over negation, as shown in (7) its prejacent will express a negative proposition resulting in a "least-likely-tonot" inference, which is, of course, equivalent to "most-likely-to" inference.

(5) $[$ Even $[(\mathrm{NOT}) \mathrm{S}]]$

(6) $\llbracket$ even $\rrbracket^{w, g}(C)(p) \Leftrightarrow \mathrm{p}$ is the least likely member of C. $\mathrm{p}^{5}$

(7) [ Even [ Kim had not invited Sam to the party ]]

- Least-likely inference: That Kim hadn't invited Sam to the party is the least-likely proposition of the form that Kim hadn't invited $X$ to the party $\Leftrightarrow$ Most-likely inference: That Kim invited Sam to the party was most-likely.

The second approach (Rooth 1985, henceforth the ambiguity theory $)^{6}$ proposes that even is lexically ambiguous. One lexical entry is the one assumed by the scope theory, which has an (in principle) unrestricted distribution. The other lexical item, however, is a Negative Polarity Item (NPI) that comes with a most-likely presupposition, (9), and is restricted in its distribution to occur below negation, (8)-(10).

(8) [NOT [ $\left.\left.\operatorname{even}_{N P I}[\mathrm{~S}]\right]\right]$

(9) $\llbracket \operatorname{even}_{N P I} \rrbracket^{w, g}(C)(p) \Leftrightarrow \mathrm{p}$ is the most likely member of C. $\mathrm{p}$

(10) [NOT [ even $_{N P I}$ [ Kim had invited Sam to the party ]]] - Most-likely inference: That Kim had invited Sam to the part is the most likely proposition of the form that Kim had invited $X$ to the party.

The prejacent of even $_{N P I}$ in (10) is a clausal constituent without negation. The scalar inference will therefore target a positive proposition, which will yield a most-likely inference since the presuppositional requirement of even ${ }_{N P I}$ demands of $p$ to be the most likely element in $C$. On the ambiguity theory, then, the inferences attributed to even in various environments are determined by a lexical specification of NPI-hood rather than by scope. Even $N P I$ appears in contexts where NPIs are licensed, and is specified to trigger a most-likely inference. In a context where an NPI would not be licensed, regular even is used, giving rise to a least-likely inference.

Both of these theories can successfully analyze the examples we have seen so far, and much continued debate attempts to distinguish them. Of interest here is that they differ in which parts of the grammar are responsible for a given inference. On the scope theory, examples like (1) carry an unambiguous least-likely inference because even always has a least-likely presupposition. On the ambiguity theory, examples like (1) are unambiguous due to an additional component of the grammar, namely a constraint on the distribution of NPIs.

The unambiguous most-likely inference in (2) can likewise be accounted for by both proposals. However, in this case, both proposals depend on an additional component of the

\footnotetext{
${ }^{5}$ Notation: $\llbracket \alpha \rrbracket=\phi . \psi$ states that the semantic value of $\alpha$ is defined only if $\phi$ and when defined $\llbracket \alpha \rrbracket=\psi$.

${ }^{6}$ See (Rullmann, 1997, 2007, a.o.).
} 
grammar, extrinsic to the lexical specification of even, to rule out ambiguity. Relying solely on the basic meaning of even as a sentential operator, which requires only that even combines with a propositional node, makes a scope position below negation (a propositional operator in its own right) in principle suitable on both theories. Thus, both theories need to appeal to a mechanism extrinsic to the lexical specification of even that prevents a leastlikely inference to surface.

Anticipating the format of our experimental material, we illustrate this point with a sentence that employs even in presubject position to the left of negation, (11). Both theories can explain the attested most-likely-to/least-likely-to-not inference by assuming that (regular) even scopes above negation, (11a). However, both theories also need to explain why a logical form where (regular) even scopes below negation, which would give rise to a least-likely-to inference, is not attested, $(11 \mathrm{~b})^{7}$.

(11) Even AMY wasn't invited to the party.

a. [ Even [ NOT [ AMY was invited to the party ] ] ]

b. * [ NOT [ even [ AMY was invited to the party ] ] ]

- Scope Theory: violates scope constraint for even

- Ambiguity Theory: Blocked by even ${ }_{N P I}$

On the scope theory (11b) is ruled out by a rather specific prohibition against interpreting even below clause-mate negation. On the ambiguity theory, a blocking principle of some sort is required to ensure that the availability of even $N P I$ preempts regular even from being inserted in this environment [e.g., because more specified lexical items, even ${ }_{N P I}$ in this case, are prioritized by principles of vocabulary insertion over less specified lexical items, non-NPI even, along the lines of Halle and Marantz (1993)].

\subsection{Predicting the Acquisition Profile of Even}

Given two possible inferences (least-likely and most-likely) and two types of environments (positive and negative, or more generally, upward and downward entailing ${ }^{8}$ ), we might expect a learner to consider four possible ways to use even. A learner who hypothesizes that even is polysemous between a most/leastlikely inference may start with all four use-patterns in the space (cf. Giannakidou, 2007 on Greek) (Table 1A). A learner who entertains only a least-likely inference associated with even (along the lines of the scope theory), however, should only consider three at the outset (Table 1B).

The acquisition path that each of these hypothetical learners takes is expected to be different because they have different starting points. When confronted with a positive even-sentence, (12), a learner who hypothesizes a uniform least-likely inference is expected to appear adult-like, because they should only detect a least-likely inference in this context. By contrast, a learner

\footnotetext{
${ }^{7}$ The precise mechanism (e.g., some form of reconstruction) by which such a logical form might be generated is not important to our purpose.

${ }^{8}$ In this paper, we abstract way from the interpretation of even in non-monotonic contexts, see e.g., Crnič, 2014.
}

TABLE 1 | Space of in principle available inferences associated with even on each theory.

\begin{tabular}{lccc}
\hline & \multicolumn{2}{c}{ Likelihood inference } \\
\cline { 3 - 3 } & Least-likely & Most-likely \\
\hline (A) AMBIGUITY THEORY OF EVEN & & $\checkmark$ \\
Sentence polarity & POS & $\checkmark$ & $\checkmark$ \\
(B) SCOPE THEORY OF EVEN & $\checkmark$ & \\
Sentence polarity & POS & $\checkmark$ & $\checkmark$ \\
\end{tabular}

who entertains a polysemous even should find sentences like (12) ambiguous between a most-likely/least-likely interpretation. The profile of this type of learner is therefore expected to be non-uniform-at-times adult-like and at-times non-adult-like depending on how they choose to resolve the ambiguity.

(12) Even AMY was invited to the party.

- Uniform least-likely even: adult-like

- Polysemous even: adult-like or non-adult-like depending on ambiguity resolution

(13) Even AMY wasn't invited to the party.

- Uniform least-likely even: adult-like or non-adult-like depending on scope

- Polysemous even: adult-like or non-adult-like depending on ambiguity resolution

When confronted with a negative even sentence, (13), however, both learners are predicted to detect an ambiguous mostlikely/least-likely inference. For the learner who posits a uniform least-likely inference, this is because even has two in principle available scope positions with respect to negation. For the polysemous even learner, they always posit these two inferences regardless of scope.

For a uniform least-likely even learner, getting to the adultlike pattern, then, amounts to ruling out ambiguity in negative environments, presumably by expunging the low scope option for even in those contexts. For a polysemous even learner, they must rule out ambiguity in both polarity environments to arrive at the adult pattern. And they must do so in a specific way: in positive environments the most-likely variant of even must be expunged while in negative environments the least-likely variant is targeted ${ }^{9}$.

These different learning profiles should be detectable in a wellcontrolled acquisition study as soon as a child has the machinery to reason about likelihood, and identifies even as a linguistic device used to convey likelihood inferences. To our knowledge, however, this has not been successfully investigated. Rather,

${ }^{9}$ There is hypothetically a third possibility, which is that a child first hypothesizes a uniform most-likely inference associated with even. The learning path of a learner with this profile is unpredictable because the child would effectively have to start over at some point by adding to their hypothesis space. 


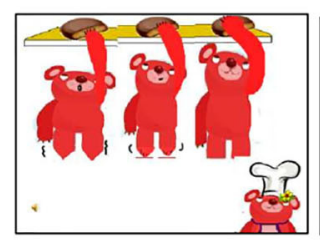

The three bears were very excited about eating the cookies. Each bear tried to reach the cookies on the shelf and managed to do so. Mama Bear said, "Even Larry was able to reach the cookie."

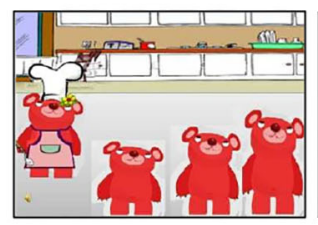

This story is about Mama Bear and her three sons. Look at the three sons. Each one has a different height. Who is the shortest one? Can you point to him? Who is the tallest one? Can you point to him?

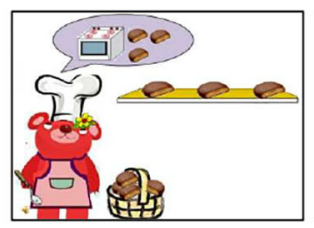

Since Mama Bear wanted to know how high her sons could reach, she put three cookies on a shelf and then asked each of them to try to reach the cookies. Mama Bear said, "I made cookies for you guys. After all of you try to reach the cookies, let's have them together."
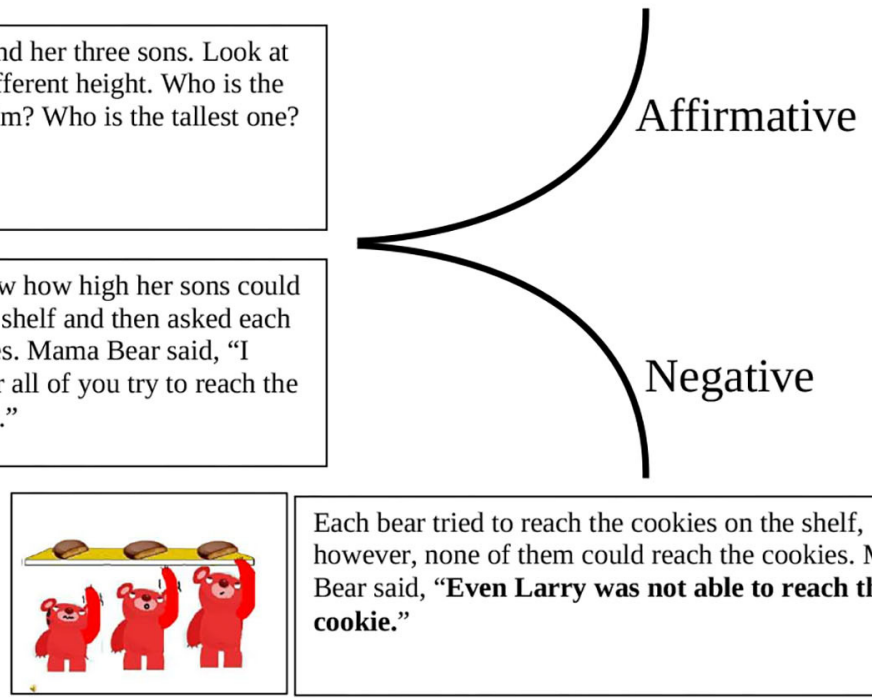

Each bear tried to reach the cookies on the shelf, however, none of them could reach the cookies. Mama Bear said, "Even Larry was not able to reach the cookie."

FIGURE 1 | Sample story setup (Kim, 2011).

existing acquisition studies of even aim to address a coarser question: do children know even? The findings of these studies have been taken to indicate that children struggle so much with even's focus and scalar properties, that it is unreasonable to think one could ever glean the specific shape of the child's hypothesis space for the likelihood inferences associated with even.

We will argue, however, that these conclusions are not wellsupported by the experimental record. Additionally, we will show that controlling for previously uncontrolled experimental factors reveals a more intricate structure of the development of the grammar and learning path of even. In particular, we uncover evidence that children as young as 4 years of age do entertain a space like those in Table $\mathbf{1}$, and moreover that they start with a space that resembles the ambiguity theory of even.

\subsection{Previous Work: Kim (2011)}

Motivation for our study, as well as inspiration for its design, is due in part to results from Kim (2011), which to our knowledge is the first systematic investigation of even in positive and negative environments in child language. Kim framed her interest in even within the context of prior studies on the acquisition of scalar implicatures. Echoing the consensus view of work in that domain, Kim argues that children as old as 5 are essentially ignorant about even. However, we find her results to be ambiguous between two interpretations: (1) children ages 45 do not detect even's scalar inferences, or (2) children ages 4-5 do detect even's scalar inferences, but nevertheless exhibit nonadult-like behavior that is invited by their developing grammar of even.

Kim (2011) conducted a comprehension experiment employing a forced-choice task, in which the experimenter tells children stories about 3 characters who are all different sizes. In each story, all of the characters are supposed to do a task, which scales in difficulty with the size of the character. At the end of the stories, either all of the characters succeed or they all fail. The end of the story is accompanied by a prompt of the form, Even $X$ was/n't able to do $Y$. With the help of a puppet, the children are asked to point to X. Figure 1 shows a sample story setup about bears reaching for cookies on a shelf, with both possible story outcomes.

Kim tested 30 children on three positive/negative story pairs of this sort, yielding 90 responses, where each "response" corresponds to a pair of responses to the positive/negative versions of each story (Table 2). There were 3 distinct response profiles corresponding to a given positive/negative pair. Some were completely adult-like ("target characters for both sentence types"), some gave opposite of adult-like responses by choosing the tallest character in positive environments and the shortest character in negative environments ("opposite characters for both sentence types"), and some uniformly chose either the rightmost or leftmost character, regardless of polarity ("always rightmost or leftmost character"). Here "both sentence types" refers to both positive and negative prompts (i.e., even $X$ was able to do Y/even $X$ wasn't able to do $Y$ ). 
TABLE 2 | Rate of responses out of different types of pragmatics for test and control sentences in children's group. From Kim (2011).

\begin{tabular}{|c|c|c|c|c|c|}
\hline & & \multicolumn{4}{|c|}{ Selection pattern } \\
\hline & & $\begin{array}{l}\text { Target characters for both } \\
\text { sentence types }\end{array}$ & $\begin{array}{l}\text { Opposite characters for } \\
\text { both sentence types }\end{array}$ & $\begin{array}{l}\text { Always rightmost or leftmost } \\
\text { character }\end{array}$ & Any characters \\
\hline \multirow{2}{*}{ Sentence type } & Test sentences & $33.3 \%(36 / 90)$ & $38.9 \%(35 / 90)$ & $\begin{array}{l}27.8 \%(25 / 90)(22.2 \% \text { for } \\
\text { rightmost, 5.6\% for leftmost) }\end{array}$ & \\
\hline & Control sentences & $20 \%(18 / 90)$ & $40 \%(36 / 90)$ & $\begin{array}{l}36.7 \%(33 / 90) \text { (26.7\% for } \\
\text { rightmost, 10\% for leftmost) }\end{array}$ & $3.3 \%(3 / 90)$ \\
\hline
\end{tabular}

In addition, Kim ran a control version of the experiment in which even was removed from the prompts. No other changes were made to the stories or the task in the control experiment. For example, in the bear story the prompt might be Larry was (not) able to reach the cookie. The control study has the interesting property that an adult would presumably struggle to find a felicitous answer to the question, "Who is Larry?", given that "Larry was (not) able to reach the cookie" is equally true of every character. Despite the pragmatic oddity of the task, and the lack of an "adult-like" target answer, Kim shows that children performed very similarly in the control experiment as they did in the main experiment with even (Table 2).

The fact that children showed low rates of adult-like responses in the even experiment, as well as the fact their responses didn't change substantially when even was removed in the control experiment, led Kim to conclude that children essentially ignore even when participating in these comprehension tasks. She therefore concludes that the children in this age group do not understand even.

We think, however, that her results are compatible with an alternative explanation and so are not compelled to accept her conclusion. Notice that in her main experiment, none of the children chose the middle character. All response profiles included one or both of the extrema, but never the middle. This is unexpected if their selection were truly random. While it is possible to conclude with Kim that this is an accidental result, or a product of the pragmatics of the task, in an unpublished manuscript, Kenyon Branan argues that this pattern could also reflect a feature of the developing grammar. In other words, it might be the case that children don't choose the middle character because they know that even triggers either a least-likely or a most-likely inference, neither of which supports picking the middle character. What they don't know, on this conjecture, are the grammatical conditions that control in the adult grammar which likelihood inference is triggered in which environment.

Looking closer at Kim's design and procedures reinforces being more cautious in interpreting her results. Note first that Kim's child participants were asked to point out both extrema characters but never the middle character during the story leading up to the even sentence. This introduces a potential bias in favor of the extrema which makes it difficult to assess whether this gap should be seen as an experimental artifact or as a reflection of a non-adult-like grammar.

Second, the specific choices Kim made in the design of her materials introduce a potential confound. Concretely, her experiment employed three story types, 2 of which involved reaching tasks, and one of which was a lifting task. For both reaching and lifting stories, the likelihood of success is directly proportional to the size of the character. Larger characters are both taller and stronger, and are thus, all else being equal, more likely to succeed at reaching something tall or lifting something heavy. An adult-like response to these story types thus amounts to choosing the smallest character in positive environments (least likely to succeed), and the largest character in negative environments (least likely to not succeed). However, this set up introduces a confound for children who showed a preference for the rightmost or the leftmost character. Such a preference could be interpreted in multiple ways. One interpretation is that children have an irrelevant preference for either the smallest or the largest character (or the rightmost or leftmost character). This interpretation is compatible with Kim's conclusion that children ignore even completely when doing the task.

However, this behavior is also explained if children are accessing likelihood inferences that are not detectable in adult language, but are present in their hypothesis space for even. If for any given example, a child is aware that both a mostand a least-likely inference is in principle available, the child might sometimes choose the most-likely character to succeed, and sometimes the least-likely character to succeed, irrespective of the polarity of the sentence. Choosing either the most- or leastlikely character uniformly in both polarity environments would amount to a right-most or left-most preference, in which case this response pattern should not be treated as evidence of naivety. In sum, then, Kim's observations are amenable to two quite different explanations and additional work is required to decide which one is on the right track.

\section{COMPREHENSION EXPERIMENT 1}

Our own comprehension experiments adopt Kim's basic setup, which we think is quite elegant and offers a very natural way of testing children's comprehension of even. We are, however, implementing a number of modifications to help overcome the aforementioned limitations in interpreting her results.

\subsection{Methods}

Experiment 1 adopts the basic format of Kim's set-up, but includes the modifications and amendments summarized below. Examples of stimuli used in Experiment 1 can be seen in Figure 2.

1. All characters are equally prominent in the story dialogue to avoid biasing children toward the extrema. Unlike Kim's 


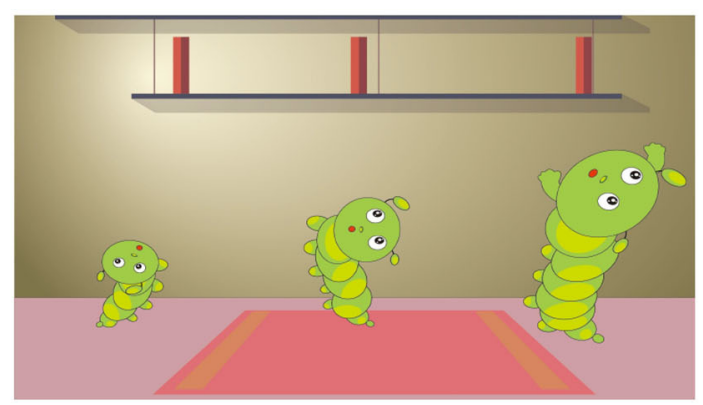

\section{"Even Jessiepillar $F$ wasn't able to reach a book!"}

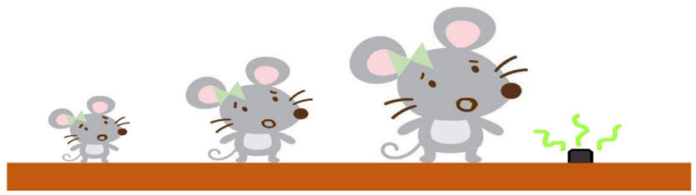

"Even Mary $F$ wasn't able to fit into the hole!"

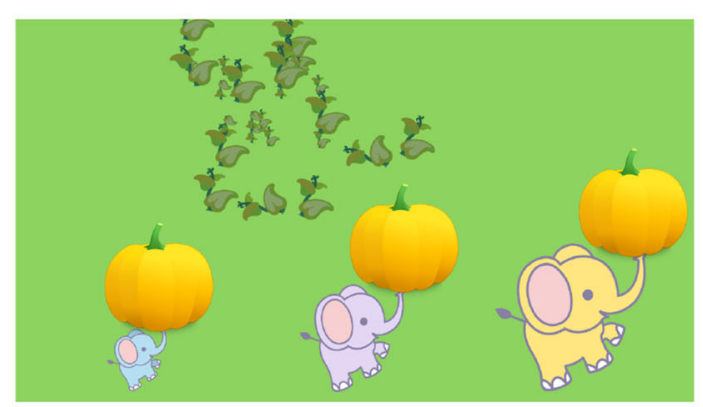

"Even Rufus $F$ was able to lift a pumpkin!"

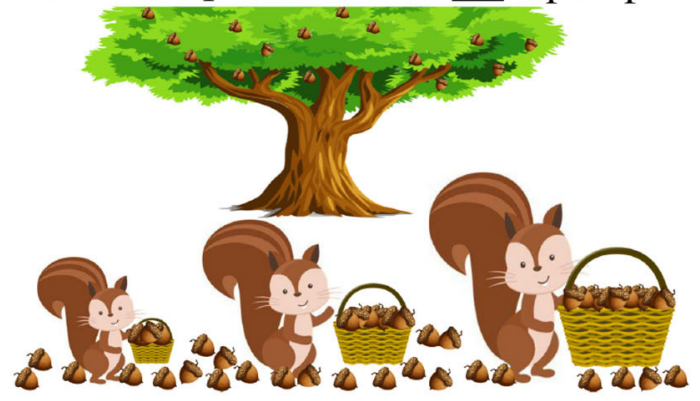

"Even Sammy $_{F}$ was able to fill her basket!"

FIGURE 2 | Four different scale types for the target stimuli: reach, lift, fit, fill.

dialogue, which singles out the extrema characters ("Who is the shortest [bear]? Who is the tallest [bear]?"), our dialogue gives equal weight to all three characters ("This story is about 3 squirrel brothers. There's a little one, a medium one, and a big one.").

2. We include filler stimuli without even where the middle character is the correct answer, both to test children's alertness and to give them an opportunity to see that the middle character is a possible answer (Supplementary Figure 1). The filler stimuli involve matching a character to an object based on attributes like color and size.

3. Children are asked to justify their responses (e.g., "How did you know that was Larry?").

4. There are two additional story types in which likelihood to succeed scales inversely with the size of the character (i.e., fitting and filling stories). This distinguishes a preference for the smallest character from a preference for the least-likely character ${ }^{10}$.

5. The age range of our study is 3-6yo to look for a possible developmental trajectory for even (in contrast with Kim's range of 4-5yo).

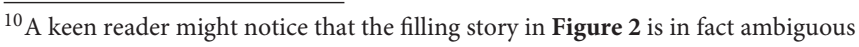
between two possible likelihood inferences: (1) likelihood of the basket to be filled up (smallest basket $=$ most-likely) vs. (2) likelihood of the character to collect enough for their basket (largest character $=$ most-likely). Indeed we will see that children and adults alike are sensitive to this ambiguity, which added noise to our initial results, and prompted a change to these stimuli in Experiment 2.
}

We recruited 91 English-speaking children ages 3;1-6;11 (mean $=5 ; 0$, age in years;months format) from Boston-area daycares, preschools, and through the Living Laboratory program at the Museum of Science, Boston. Three subjects were excluded from the analysis either because they did not complete the task, or because their responses on the control items (as well as justifications) suggested they were not actively participating in the task.

In total, the experiment consisted of 4 filler stimuli and 8 target stimuli: 4 positive target stimuli (1 each for reach/lift/fit/fill) and 4 negative target stimuli ( 1 each for $\mathrm{reach} / \mathrm{lift} /$ fit/fill). The experimental items were blocked by polarity of the even sentences (NEG vs. POS) and presented in one of two orders (NEG-first vs. POS-first). Children who were assigned to the NEG-first order heard all of the negative stimuli before hearing any positive stimuli, and vice versa.

As a control, we ran a version of this experiment with 68 adult subjects on Mechanical Turk. We slightly modified the stimuli from the child study for use on IBEX (Drummond, 2012), creating an introductory slide which introduced the characters and the situation, and a question slide, which introduced the even sentence and asked the participant to identify the named character. Participants were given 10s to respond, starting immediately after the question slide was displayed. IBEX recorded question responses, reaction times, as well as justifications. We followed two exclusion criteria: (1) we excluded participants who incorrectly answered more than one filler item, (2) we excluded participants who gave the same answer (e.g., 
the middle character) throughout the entire study. After these exclusions, 56 subjects remained.

Data were analyzed using the MCMCglmm package in R (Hadfield, 2010) with a mixed-effects multinomial logistic regression. We took response type as the dependent variable, and modeled the fixed effect of polarity and age group \{3yo, 4 yo, 5yo, 6yo . Additionally, we modeled a random intercept by subject and by story type. We opted to use a Bayesian approach to our data analysis for two main reasons. First, Bayesian approaches make it relatively easy to specify hierarchical models, such as the multinomial mixed-effects model deployed here, as compared to frequentist data analysis. Second, Bayesian models with maximal random effects structures converge with less data than frequentist models. Four chains were generated per model, and convergence was tested across these chains using the Gelman-Rubin diagnostic (Gelman and Rubin, 1992; Plummer et al., 2006), as well as by visual inspection of the posterior distributions. Credible Intervals were calculated at $95 \%$ with Highest Posterior Density intervals ${ }^{11,12,13,14}$.

\subsection{Results}

In our original experimental design, polarity (NEG vs. POS) was a within-subject condition and order (NEG-first vs. POS-first) was a between-subject factor. However, our models failed to converge with order as a factor. Because of this, we chose to only take data from the first four target items from each subject, removing order as a condition, and turning polarity into a between-subject factor. This way we can be sure that our reported results on the interaction between response-type and polarity is not affected by possible within experiment learning or other order effects throughout the experiment ${ }^{15}$.

\footnotetext{
${ }^{11}$ For further background on the use of Bayesian methods in the context of linguistics, see (Nicenboim and Vasishth, 2016).

${ }^{12}$ Credible Intervals, used in Bayesian analysis, are intervals which contain a certain percentage (in this paper, 95\%) of the values in the posterior distribution for an unobserved parameter. For instance, a 95\% Credible Interval of [10\%, 22\%] indicates that we can be $95 \%$ certain that the value of a parameter falls between $10 \%$ and $22 \%$. In this paper, we follow a basic decision rule to take the $95 \%$ Credible Interval as an indication of whether a given value can be inferred to be a possible "true" value of a parameter (Kruschke et al., 2012). Thus, the CI here serves a similar role to the $p$-value used in frequentist statistics, in giving us a criterion to reject or accept the null hypothesis.

${ }^{13}$ Highest Posterior Density is a method for selecting Credible Intervals which, as might be expected, involves selecting the highest density intervals within the posterior distribution. Note that because HPD intervals are chosen by density of the posterior distribution, they will not center around the mean.

${ }^{14}$ All code used for the analyses in this paper can be found here: https://github. com/MITLanguageAcquisitionLab/even.

${ }^{15}$ Though our experimental design included filler items for the purpose of potentially excluding inattentive participants, a problem with one of them prevented us from using these filler items as grounds for excluding subjects in our analysis of Experiment 1. Our original exclusion criteria would have excluded children who answered more than 1/4 filler items incorrectly (allowing for occasional but not systematic lapses in judgment). However, our child participants systematically struggled with one of the filler items, even when it was clear that they were attentive throughout the experiment. It was therefore clear that we should remove that item from our consideration for exclusion. However, doing so increased the likelihood of exclusion from $2 / 4$ to $2 / 3$, which we felt was too significant a difference to implement without probable cause. We therefore chose to be maximally inclusive of all subjects who completed the study.
}

The results of our experiment differ from Kim's in several ways. The children that we tested offered three types of responses, which we call adult-like, middle, and opposite, corresponding to which character they chose. While Kim's results had no middle responses, in our study, subjects ages 3-5 did sometimes choose the middle character for target items. We also see a steady increase in the number of adult-like responses across age, and a stable number of opposite responses. Figure 3 summarizes the results of Experiment 1.

Figure 3B shows the rate of adult-like responses across age, split by polarity. What we find is that this rate increases much more quickly in negative environments than in positive environments. At age 4, there is a clear difference between the positive and negative environments with respect to adultlike responses $([-3 \%, 74 \%])$. The profile of even comprehension in negative environments is quite stable across ages 4-6 (4yo: [56\%, 98\%], 5yo: [56\%, 97\%], 6yo: [63\%, 99\%]) while adultlike behavior lags in positive environments at age 4, rising steadily until age 6 (4yo: [12\%, 74\%], 5yo: [48\%, 100\%], 6yo: $[73 \%, 100 \%])$.

A complementary trend can be seen in the rate of middle responses (Figure 3C), which given that they are never invited by the grammar are an indication of confusion. We see that the number of middle responses remains low, decreasing over time (3yо: [0\%, 42\%], 4yo: [1\%, 27\%], 5yo: [0\%, 30\%], 6yo: [0\%, 3\%]).

Looking at the last response type, opposite responses, reveals a different pattern from both adult-like and middle responses. Opposite responses are stable across the four age groups, hovering at approximately 20-25\% (Figure 3D) (3yо: [0\%, 49\%], 4yo: [5\%, 38\%] 5yo: [0\%, 17\%], 6yo: [0\%, $22 \%]$ ). Additionally, we do not see a polarity effect for opposite responses.

We also asked children to justify their answers and coded their responses as scalar, random or none to reflect whether their justifications contained evidence of scalar reasoning. In general, all justifications that referred to the size of the characters were coded as "scalar," and all responses were coded jointly by the two first authors. Some sample justifications that we coded as "scalar" include: "Because it's rare that a tiny thing can lift a big thing," "teeny one," "small mouses can usually fit," "because it's the biggest," etc.

Children also often provided reasons that did not reference a discernible scale, which we coded as "random." Some sample "random" justifications include: "look at the pink bunny!", "because I just knew it," "he's two (years old)," "that one has a little bow," etc. Some children were too shy to offer a justification, or stated that they didn't know why they chose a particular character, in which case we coded their responses as "none."

Table 3 summarizes the number of justifications of each type that were provided for each response pattern. Notably, scalar justifications were predominantly offered for adult-like and opposite responses, while random justifications were the most frequent justification type for middle responses.

The overall results of the adult control study appear in Table 4 . The adults, as expected, predominantly pick the leastlikely character in positive environments (88\%) and the mostlikely character in negative environments $(82 \%)$. This shows a 


\section{A \\ Response types by age group}

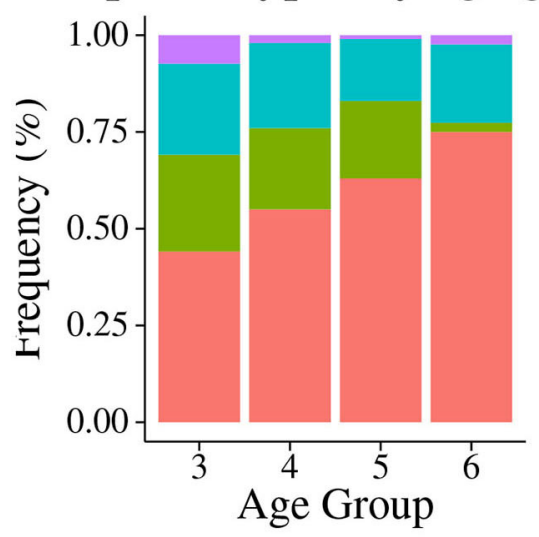

c Middle by age group

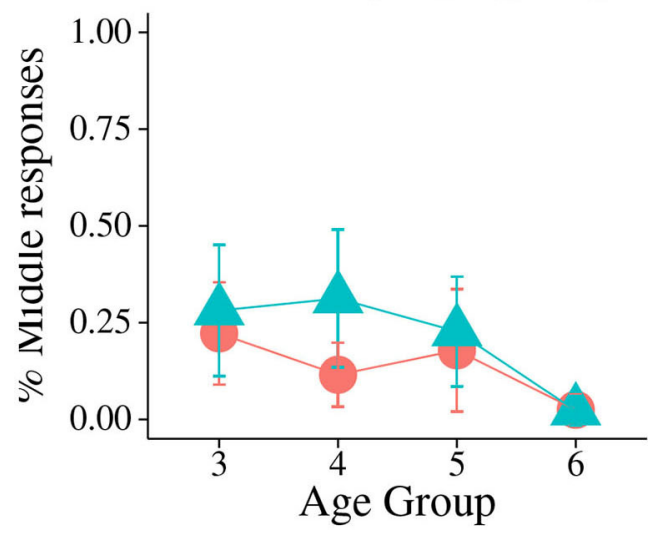

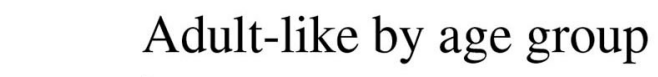
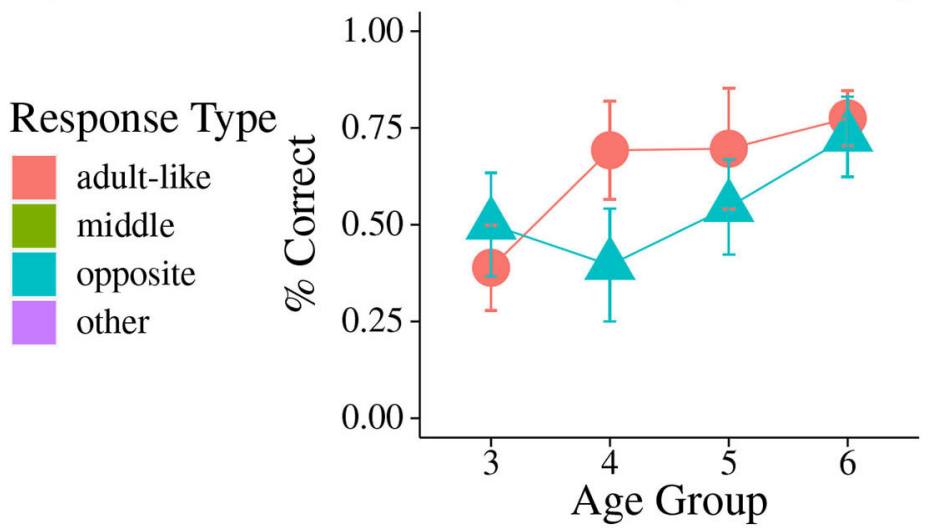

polarity

NEG

POS
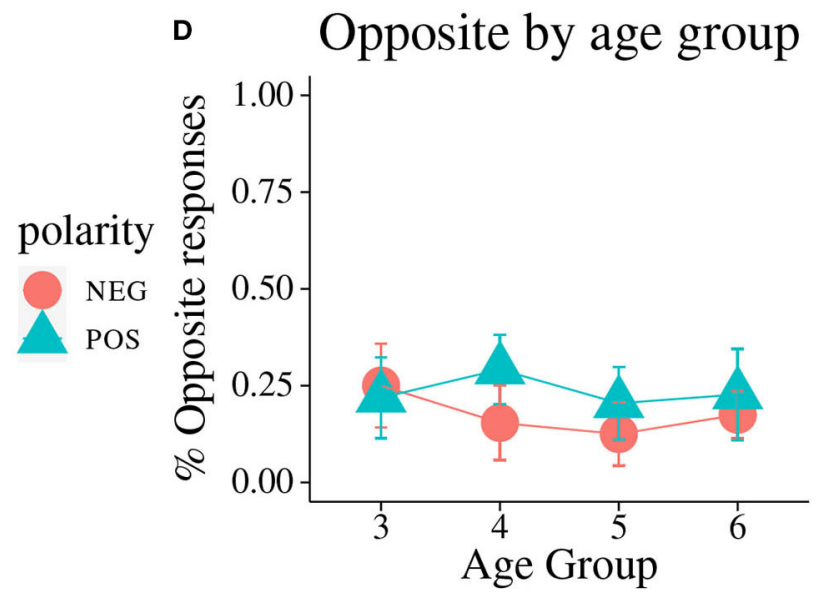

polarity

NEG

POS

FIGURE 3 | Results for Experiment 1. (A) Rate of response types by age group. (B) Rate of adult-like responses by age group and polarity. (C) Rate of middle responses by age group and polarity. (D) Rate of opposite responses by age group and polarity.

TABLE 3 | Experiment 1: Justifications for each response type by age.

\begin{tabular}{|c|c|c|c|c|c|c|c|}
\hline Response type & Justification type & Age group: & 3 & 4 & 5 & 6 & Total \\
\hline & None & & 17 & 16 & 9 & 12 & 54 \\
\hline \multirow[t]{3}{*}{ Adult-like } & Random & & 0 & 4 & 7 & 6 & 17 \\
\hline & Scalar & & 13 & 35 & 47 & 45 & 140 \\
\hline & none & & 15 & 9 & 5 & 1 & 30 \\
\hline \multirow[t]{3}{*}{ Middle } & Random & & 2 & 9 & 11 & 1 & 23 \\
\hline & Scalar & & 0 & 3 & 4 & 0 & 7 \\
\hline & none & & 10 & 10 & 3 & 1 & 24 \\
\hline \multirow[t]{2}{*}{ Opposite } & Random & & 3 & 3 & 2 & 1 & 9 \\
\hline & Scalar & & 3 & 9 & 11 & 15 & 38 \\
\hline
\end{tabular}

slight asymmetry in favor of positive stimuli-however, it is not statistically significant $^{16}$.

\footnotetext{
${ }^{16} \mathrm{~A}$ similar asymmetry in the same direction can be seen in terms of response times, with negative stimuli taking longer than positive stimuli, but this difference is again not statistically significant.
}

TABLE 4 | Experiment 1, Adult control: Rates of response types by polarity.

\begin{tabular}{llcc}
\hline Response type & Polarity & \% of responses & Std.dev (\%) \\
\hline \multirow{2}{*}{ Adult-like } & POS & 88 & 32 \\
& NEG & 82 & 38 \\
Middle & POS & 0 & 0 \\
& NEG & 2 & 14 \\
Opposite & POS & 12 & 32 \\
& NEG & 16 & 37 \\
\hline
\end{tabular}

\subsection{Analysis and Discussion}

We want to draw attention to several features of these results, some of which resolve open questions in Kim's experiment, and some of which raise new ones.

First, we argue that these results refute Kim's conclusion that children ages $4-5$ show no evidence of comprehending even. Our results show a clear upward trajectory in the rate of adult-like responses between the ages 3-6, as seen in Figure 3A. 
TABLE 5 | Summary of statistical analysis for experiment 1: (A) Mean differences between age groups in rate of adult-like, middle, and opposite responses, for all data (B) Mean differences between age groups in rate of adult-like, middle, and opposite responses, for just negative stimuli. (C) Mean differences in rate of justification types by response type.

\begin{tabular}{|c|c|c|c|c|c|c|c|c|c|}
\hline \multirow{2}{*}{$\begin{array}{l}\text { Age Group vs. } \\
\text { Age Group }\end{array}$} & \multicolumn{3}{|c|}{4 yo } & \multicolumn{3}{|c|}{5 уо } & \multicolumn{3}{|c|}{$6 y o$} \\
\hline & & Mean & Cl & & Mean & Cl & & Mean & Cl \\
\hline \multicolumn{10}{|l|}{ (A). ALL DATA } \\
\hline & Adult-like & 13 & $-11 ; 39$ & Adult-like & 33 & $-2 ; 67$ & Adult-like & 37 & $10 ; 66$ \\
\hline \multirow[t]{3}{*}{ 3yo } & Middle & -6 & $-34 ; 16$ & Middle & -12 & $-47 ; 21$ & Middle & -17 & $-43 ; 0$ \\
\hline & Opposite & -6 & $-26 ; 13$ & Opposite & -21 & $-44 ;-1$ & Opposite & -20 & $-44 ; 2$ \\
\hline & & & & Adult-like & 20 & $-10 ; 48$ & Adult-like & 24 & $2 ; 47$ \\
\hline \multirow[t]{3}{*}{4 yo } & & & & Middle & -5 & $-30 ; 22$ & Middle & -11 & $-28 ; 0$ \\
\hline & & & & Opposite & -15 & $-33 ; 0$ & Opposite & -13 & $-33 ; 4$ \\
\hline & & & & & & & Adult-like & 4 & $-13 ; 29$ \\
\hline \multirow[t]{2}{*}{5 yo } & & & & & & & Middle & -6 & $-28 ; 3$ \\
\hline & & & & & & & Opposite & 1 & $-9 ; 13$ \\
\hline \multirow{2}{*}{$\begin{array}{l}\text { Age Group vs. } \\
\text { Age Group }\end{array}$} & & 4уо & & & 5 yo & & & 6 ууо & \\
\hline & & Mean & $\mathrm{Cl}$ & & Mean & Cl & & Mean & $\mathrm{Cl}$ \\
\hline \multicolumn{10}{|c|}{ (B). ONLY DATA FROM NEGATIVE STIMULI } \\
\hline & Adult-like & 29 & $0 ; 59$ & Adult-like & 29 & $0 ; 60$ & Adult-like & 34 & $5 ; 65$ \\
\hline \multirow[t]{3}{*}{ 3yo } & Middle & -13 & $-48 ; 11$ & Middle & -10 & $-47 ; 16$ & Middle & -17 & $-50 ; 1$ \\
\hline & Opposite & -17 & $-44 ; 7$ & Opposite & -19 & $-46 ; 4$ & Opposite & -17 & $-45 ; 8$ \\
\hline & & & & Adult-like & 0 & $-22 ; 22$ & Adult-like & 5 & $-15 ; 27$ \\
\hline \multirow[t]{3}{*}{4 yo } & & & & Middle & 2 & $-14 ; 22$ & Middle & -5 & $-19 ; 3$ \\
\hline & & & & Opposite & -3 & $-20 ; 13$ & Opposite & 0 & $-18 ; 18$ \\
\hline & & & & & & & Adult-like & 5 & $-17 ; 28$ \\
\hline \multirow[t]{2}{*}{5 yo } & & & & & & & Middle & -7 & $-25 ; 2$ \\
\hline & & & & & & & Opposite & 3 & $-15 ; 21$ \\
\hline \multirow{2}{*}{\multicolumn{2}{|c|}{$\begin{array}{l}\text { Response Type vs. } \\
\text { Response Type }\end{array}$}} & & & Adult-like & & & Opposite & & \\
\hline & & & & Mean & Cl & & Mean & $\mathrm{Cl}$ & \\
\hline \multicolumn{10}{|c|}{ (C). MEAN DIFFERENCE IN RATE OF JUSTIFICATION TYPES BY RESPONSE TYPE } \\
\hline & & & None & -57 & $-89 ;-19$ & None & -57 & $-90 ;-18$ & \\
\hline \multirow[t]{3}{*}{ Middle } & & & Random & -8 & $-29 ; 2$ & Random & -6 & $-28 ; 6$ & \\
\hline & & & Scalar & 63 & $26 ; 94$ & Scalar & 62 & $23 ; 93$ & \\
\hline & & & None & 1 & $-24 ; 25$ & & & & \\
\hline \multirow[t]{2}{*}{ Opposite } & & & Random & -2 & $-8 ; 2$ & & & & \\
\hline & & & Scalar & 1 & $-24 ; 27$ & & & & \\
\hline
\end{tabular}

Cells highlighted in green indicate a significant difference (i.e., not centered around 0).

Furthermore, our results provide a clear indication as to when children begin to reliably exhibit sensitivity to the scalar properties of even. Given that there are three possible response types, chance behavior should be $33 \%$. While 3 yos do not give adult-like responses at an above chance rate $([17 \%, 83 \%])$, starting at 4 years old, children provide adult-like responses at well above chance, nearing adultlike levels by age 6 (4yo: [45\%, 89\%], 5yo: [60\%, 100\%], 6yo: $[76 \%, 100 \%])$.

An analysis of the mean difference between the rate of adult-like, middle and opposite responses across ages shows that by age 6 , the rate of adult-like responses has increased significantly since ages $3-4$, while the rate of middle responses has decreased significantly (Table 5A). These results reinforce our claim that children progressively acquire an adult-like understanding of even.

Looking just at the progression of adult-like responses in negative contexts, we see that the only significant difference is between the 3yo on the one hand, and the 4-, 5-, and 6yo on the other (Table 5B). There is no significant difference between the rate at which 4 yo provide adultlike responses to negative stimuli and the rate at which 5and 6yo do, suggesting that children acquire negative even sentences earlier than positive ones. This result contradicts the expectation that negation adds a computational cost. Not only are children unphased by the addition of negation, but they 
appear to have an easier time deducing the right inference in its presence.

Looking at adults in the control study, we do not see a polarity effect. There is no significant difference in rates of different response types between positive and negative stimuli.

Adults and children both show two types of error behavior, with a higher rate of opposite responses. In adults, the rate of middle responses is extremely low, but the rate of opposite responses is nearly as high as the rate of opposite responses in $6 \mathrm{yo}^{17}$.

The contrasting behavior of middle vs. opposite responses in both adults and children seems to indicate that the learning trajectory for even contains a stage at which elements of the grammar of even are already in place, but are not yet fully adultlike. The fact that middle responses disappear over time but opposite responses are stable through age 6 suggests that opposite responses are principled at this stage, while middle responses are not. In sum, we propose to analyze middle responses as indications of genuine confusion, while opposite responses should be analyzed as licensed by the developing grammar of even. Moreover, given that they are detectable at the earliest stages where children exhibit a stable appreciation of even, we propose to analyze them as an indication of what the initial hypothesis space for even looks like, Table $\mathbf{1 A}$.

The decrease in middle responses over time correlates with the increase in adult-like behavior, which supports our view of middle responses as a measure of confusion. As children become more adult-like, they become less confused. We suggest that by contrast, opposite responses are not an indicator of confusion and thus do not decrease noticeably as adult-like behavior increases. Notice that they persist to some extent even in adults.

This characterization of the middle and opposite responses is supported by the justifications that children provided for each response type. Modeling the rate of justification types by response type shows that children provide significantly more scalar justifications to adult-like and opposite responses as compared to middle responses, as seen in Table 5C. Furthermore, there is no significant difference in the rate of scalar justifications between adult-like and opposite responses. The stability of opposite responses throughout our above-chance-performing age ranges (4-6yos), combined with how well-reasoned their justifications are, suggests that children know that even is associated with a space like Table $\mathbf{1 A}$.

Recall from section 2 that the in principle space of inferences associated with even on the adult grammar included opposite inferences, which motivated the notion of the hypothesis space explored here. This prediction is readily borne out in our adult study; adults essentially only make errors in the form of opposite responses, which is expected given their grammar. The exciting finding from our child study is that children also favor this error behavior over other potential error patterns, suggesting

\footnotetext{
${ }^{17}$ Just as in the child study, we asked adults to justify their responses. Unsurprisingly since almost all errors were opposite responses, all of the justifications were scalar except for two participants that chose the middle character and wrote Just a random guess and I don't know, respectively.
}

that they too access a space like Table 1A. That said, our results contain some unexplained noise that merits further discussion. We therefore conducted a second comprehension study, whose main purpose was to investigate the potential sources of noise and their impact on our results.

One major source of noise in our data was the amount of variation in adult-like behavior across the different story types. In particular, the filling stories were accompanied by more non-adult-like responses than the other stories (Supplementary Figures 2, 3).

A clue for this pattern comes from the justifications provided by children and adults. Specifically, our subjects frequently indicated that there was a salient alternative interpretation of the filling stories. On this alternative interpretation, apparent "opposite" responses are actually adult-like, suggesting that our initial reported rates of error responses were actually somewhat inflated.

In the filling stories, the smallest character should have the easiest time filling their basket because their basket is the smallest. On an alternative construal, however, the smallest character might have the most difficulty collecting the requisite number of acorns (because of their age, or general inexperience) necessary to fill their basket. Several adult and child subjects interpreted the filling stories on the latter characterization, providing wellreasoned justifications like those in $(14)^{18}$. (14) Adult-like justifications for opposite responses in
fillingstories

a. "The first squirrel is Sammy because the youngest one would probably have the hardest time filling his basket and I assume the smallest squirrel to be the youngest." (adult)

b. "because even though his basket is little he can even fit a lot of acorns in there" (child)

Experiment 2 therefore includes new stimuli to replace the ambiguous filling stories, in order to eliminate this source of noise.

An additional concern about our experimental design pertains to the role of the ability modal in the prompts. Recall that our test subjects were asked to respond to prompts of the form Even $X_{F}$ was/n't able to do $Y$. This element of the design was inherited from Kim's experiment for the purpose of attempting to replicate her results. However, now that we have failed to replicate her results, we return to this feature of the design with some scrutiny. Of interest is the fact that ability is itself a gradable notion. To interpret a sentence with an ability modal, one must therefore have access to a kind of scalar reasoning ${ }^{19}$. A question we should ask, then, is whether the overt expression of ability accounts for any of the children's behavior on its own. To investigate the

\footnotetext{
${ }^{18}$ Note that one of the children used even in an adult-like manner to justify the opposite response (14b).

${ }^{19}$ See Greenberg, 2015, anticipated by Rullmann (2007), for an account of even that replaces the likelihood scale with a scale introduced by a (contextually salient) degree predicate.
} 


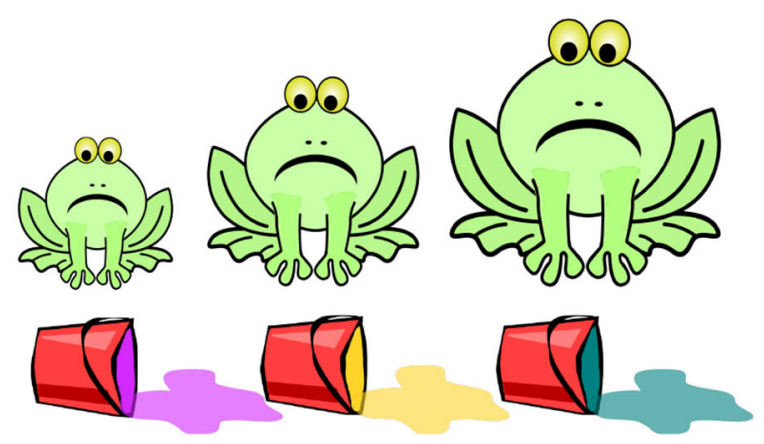

"Even Frida $F$ has spilled her paint!"

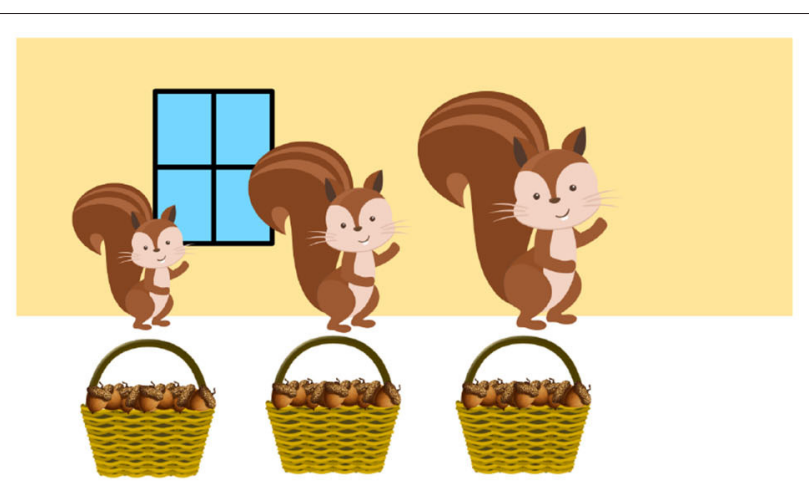

"Even Sammy ${ }_{F}$ hasn't spilled her basket!"

FIGURE 4 | Spilling stimuli.

relevance of this possible confound, we removed any overt scalar notions from our stimuli in Experiment $2^{20}$.

\section{COMPREHENSION EXPERIMENT 2}

\subsection{Methods}

Experiment 2 contains two large scale changes to the stimuli from Experiment 1: the removal of the ability modal in the prompts, (15), and the replacement of the filling stories with spilling stories (Figure 4).

(15) Sample prompts from Experiment 2 (ability modal removed)
a. Even Benny $F$ has gotten an apple!
b. Even Franky $y_{F}$ hasn't gotten the socks on!

The spilling stories are about likelihood to spill/drop something heavy. Like the fitting stories, height is inversely proportional to likelihood to spill. The smallest character is the most likely to be the weakest, and therefore the most likely to drop or spill the heavy bucket/basket.

These two changes combined are expected to avoid the problems of the previous filling stories because they remove the ambiguity about whether the likelihood scale should refer to the abilities of the characters vs. properties of their baskets/cups.

In order to make our data comparable to Experiment 1, we made polarity a between factor. Each subject evaluated eight target stimuli and four filler items, where all target stimuli were of the same polarity environment. This doubles the number of data points for each polarity condition.

\footnotetext{
${ }^{20} \mathrm{In}$ fact, we already have some reason to believe that the scalar properties of be able do not play a role in opposite responses. First, opposite responses are present in our adult data. While it is possible that some adults ignore even entirely in our experiment, this seems less likely. Additionally, we attempted to run a version of Experiment 1 with children where we did not pronounce even (e.g., Jessiepillar was able to reach the shelf.). We ultimately stopped the experiment because most of the children became very confused and didn't want to finish the task. This suggests that children who provide opposite responses are indeed sensitive to the presence of even.
}

We presented these stimuli to subjects in either of two orders, one in which the story types proceeded as fit, reach, lift, spill, and the other in reverse order. We collected data from 80 children, ages 3;1-5;11 (mean = 4;7). Unlike in Experiment $1($ see fn. 15), where children systematically struggled with a particular filler item, no such difficulty was apparent in any of the modified filler items in Experiment 2. Thus, we were able to use performance on these filler items as an exclusion criterion in Experiment 2. Subjects who failed to correctly answer at least 3 out of 4 filler questions were excluded from our results (14 exclusions), as were those whose justifications and behavior during the study suggested that they were not paying attention to the task (4 exclusions). After exclusions, 62 subjects remained. The remaining data is evenly distributed by polarity, block order, and age group, with five or six subjects per age group/polarity/order combination. We focused on these earlier years because two interesting effects apparent in Experiment 1 either disappeared or stabilized by age 6: (1) the stable preference for opposite responses over middles responses, and (2) the polarity asymmetry in the rate of adult-like responses.

As with Experiment 1, we performed an adult control study with 85 participants on Mechanical Turk with amended stimuli on IBEX (see section 3.1 for information on the changes made). After exclusion criteria were applied, data from 60 participants remained.

Data for the child and adult experiments were again analyzed using a mixed-effects multinomial logistic regression. We modeled the fixed effect of polarity and age group as well as order (forward and reverse). Additionally, we modeled a random intercept by subject and a random intercept and slope by story type. See section 3.1 for additional information on the statistical analysis.

\subsection{Results}

Figure 5A summarizes the rate of each response type by age for Experiment 2. The rate of adult-like responses again increases steadily with age (3yo: $[8 \%, 62 \%]$, 4yo: [53\%, 91\%], 5yo: [79\%, $100 \%]$ ), while middle responses decrease steadily with age (3yo: [11\%, $81 \%]$, 4yo: [0\%, $22 \%]$, 5yo: [0\%, $3 \%])$. We also see a fairly 


\section{A \\ Response types by age group}

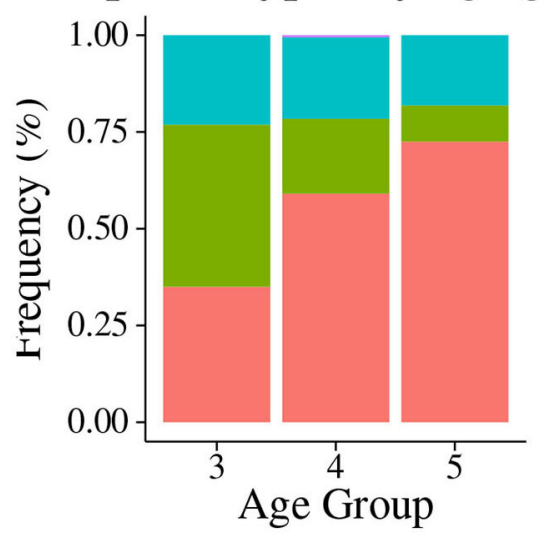

c Middle by age group

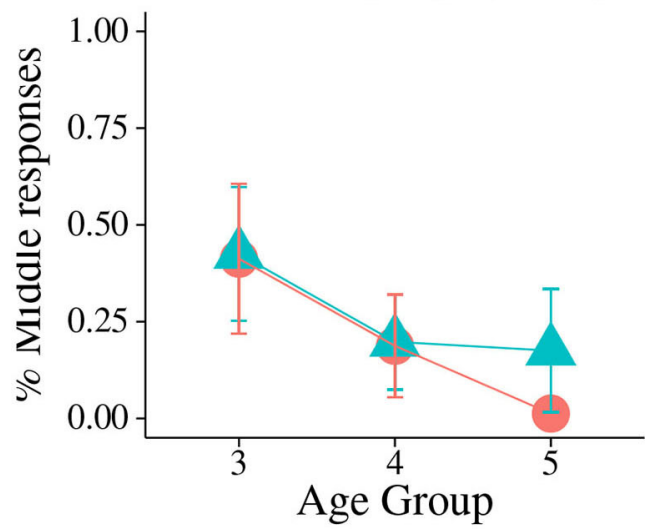

B
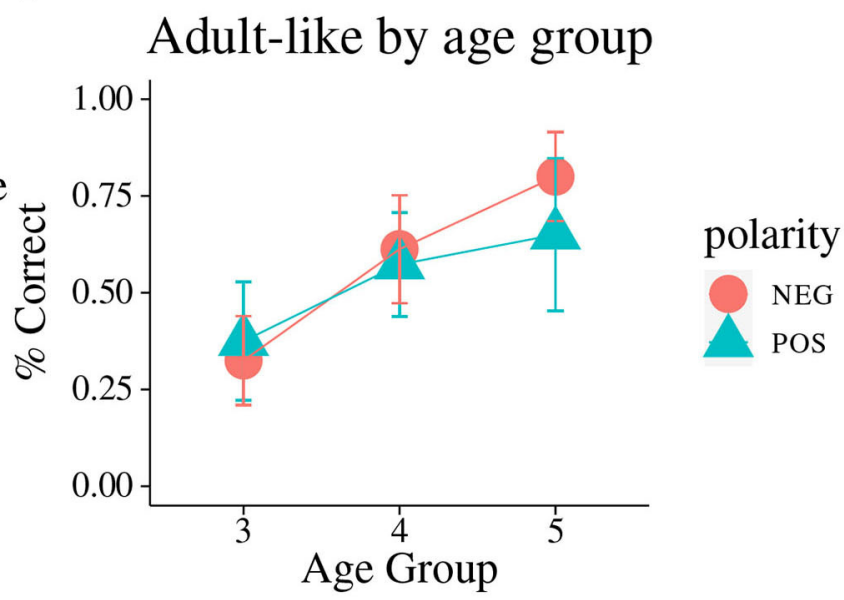

D Opposite by age group
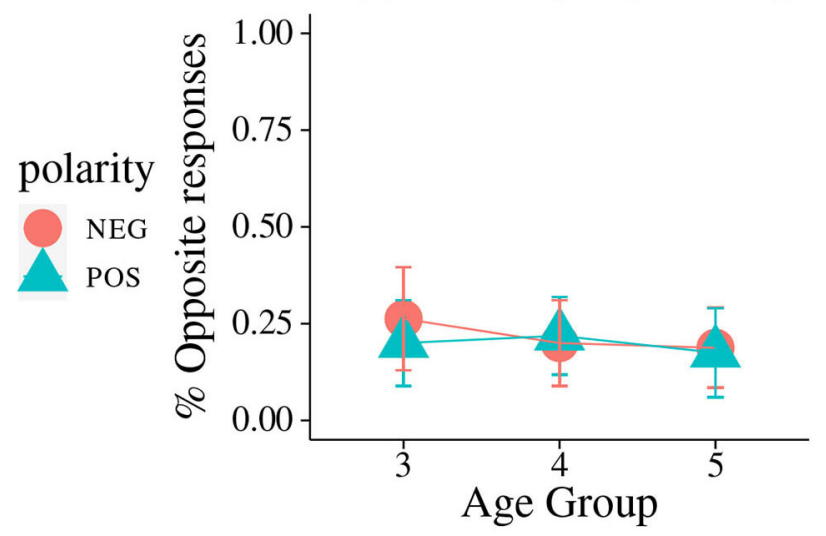

polarity

NEG

POS

FIGURE $\mathbf{5}$ | Results for Experiment 2. (A) Rate of response types by age group. (B) Rate of adult-like responses by age group and polarity. (C) Rate of middle responses by age group and polarity. (D) Rate of opposite responses by age group and polarity.

stable population of opposite responses (3yo: [3\%, 37\%], 4yo: [5\%, 33\%], 5yo: [0\%, 18\%]).

Looking at each response type individually by polarity reveals that the polarity asymmetry from Experiment 1 is far less pronounced. The rate of adult-like responses is roughly equal between the two polarity conditions in 4yos, which was where the asymmetry was most pronounced in Experiment 1. 5yos do perform noticeably better in negative environments than positive environments, but this result is not statistically significant (Figure 5B). The same pattern holds for middle responses (Figure 5C). There is no polarity asymmetry at ages 3 or 4 , but a slight asymmetry becomes visible at age 5 . Opposite responses show no sensitivity to polarity, and are even more stable than in Experiment 1 (Figure 5D).

Finally, the justifications for each response type, coded as scalar/random/none, are summarized in Table 6. As in Experiment 1, scalar justifications were offered far more for adult-like and opposite responses than for middle responses. Justifications for middle responses were most often random.

A comparison of adult-like behavior across each of the story types shows that there is less variation across items
TABLE 6 | Experiment 2: Justifications offered for each response type by age.

\begin{tabular}{|c|c|c|c|c|c|c|}
\hline Response type & Justification type & Age group: & 3 & 4 & 5 & Total \\
\hline & None & & 27 & 27 & 8 & 62 \\
\hline \multirow[t]{3}{*}{ Adult-like } & Random & & 6 & 13 & 4 & 23 \\
\hline & Scalar & & 23 & 64 & 104 & 191 \\
\hline & None & & 47 & 15 & 1 & 63 \\
\hline \multirow[t]{3}{*}{ Middle } & Random & & 16 & 13 & 8 & 37 \\
\hline & Scalar & & 4 & 6 & 6 & 16 \\
\hline & None & & 18 & 10 & 6 & 34 \\
\hline \multirow[t]{2}{*}{ Opposite } & Random & & 7 & 6 & 2 & 15 \\
\hline & Scalar & & 12 & 21 & 21 & 54 \\
\hline
\end{tabular}

compared to Experiment 1 for both adults and children (Supplementary Figures 4, 5). Most notably, the profile of responses is no longer substantially different for spilling stories than for the other story types.

The results of the adult control study are summarized in Table 7. As in Experiment 1, adults were slightly less error-prone 
TABLE 7 | Experiment 2, Adult control: Rates of response types by polarity.

\begin{tabular}{llcc}
\hline Response type & Polarity & \% of responses & Std.dev (\%) \\
\hline \multirow{2}{*}{ Adult-like } & POS & 95 & 21 \\
& NEG & 91 & 29 \\
Middle & POS & 3 & 17 \\
& NEG & 1 & 11 \\
Opposite & POS & 2 & 13 \\
& NEG & 8 & 27 \\
\hline
\end{tabular}

TABLE 8 | Experiment 2: Mean differences in (A): the rate of adult-like, middle, and opposite responses, and $\mathbf{( B )}$ : the rate of justification types (none, random, and scalar) by response type.

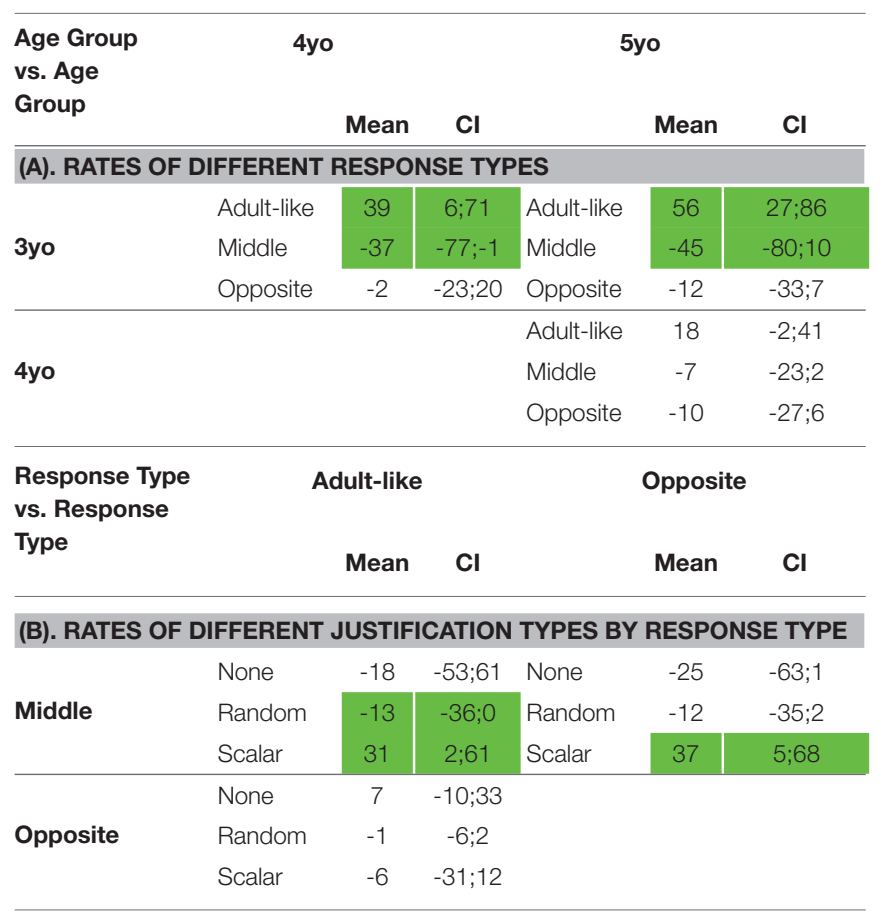

Cells highlighted in green indicate a significant difference (i.e., not centered around 0).

in positive environments ${ }^{21}$. However, this asymmetry is, again, not statistically significant.

\subsection{Analysis and Discussion}

Experiment 2 confirms the main finding from Experiment 1, namely that children begin to comprehend even earlier than Kim (2011) suggests. Once again, 3yos do not choose the adult-like response at an above chance rate [8\%,62\%], while 4yos [53\%, $91 \%]$ and 5 yos $[79 \%, 100 \%]$ perform at well above chance.

Looking at mean differences between age groups, 4- and 5 -year-olds give adult-like responses at a significantly higher rate than 3-year-olds (Table $\mathbf{8 A}$ ). However, opposite responses remain steady throughout age groups.

The opposite responses show essentially the same profile in Experiment 2 as they did in Experiment 1. Many of the children

\footnotetext{
${ }^{21}$ As in the adult control version of Experiment 1, there is also a non-statistically significant polarity asymmetry in terms of reaction time.
}

gave opposite responses, including the older ones, and these were often accompanied by scalar justifications. The middle responses, on the other hand, showed some sensitivity to age and polarity, and were primarily given random justifications, Table 8B.

Experiment 2 was successful in that it replicated these results from Experiment 1 with substantially less noise. The justifications indicate that both our adult and child participants interpreted even primarily based on the size of the characters and not based on any less obvious criteria such as perceived age or general competence. Supplementary Figures 4, 5 in the Supplementary Material show that story type also no longer correlates with any particular error pattern.

Experiment 2 also revealed that the polarity asymmetry in child comprehension observed in Experiment 1 was detectable in Experiment 2 only as a trend that did not reach statistical significance. 5yos, in particular, exhibited an advantage for negative environments on adult-like comprehension similar to that observed in Experiment 1 for 4yos. Determining what might be responsible for the similarities and differences of this environmental effect across the two experiments is unclear to us and deserves further investigation.

Interestingly, opposite responses in our adult control exhibit a noticeable sensitivity to polarity. Specifically, opposite responses are observed primarily in negative environments while positive environments generated vanishingly few ${ }^{22}$. After filtering out answers associated with justifications that indicated guessing or another interpretation of the story, there were 10 opposite responses observed in negative environments, but only 2 opposite responses in positive environments ${ }^{23,24}$.

To summarize, both Experiments 1 and 2 have enabled us to identify two distinct error profiles in adults and children. Middle responses indicate simple confusion or inattention. Opposite responses appear to be licensed by the grammar.

An important difference between adult and child error behavior is the polarity sensitivity of opposite responses. Children offered opposite responses in both positive and negative environments, suggesting that they access a space of inferences like that in Table 1A. By contrast, adults basically only offered opposite responses in negative contexts, suggesting that they access a space like that in Table $\mathbf{1 B}$.

\section{CORPUS STUDIES}

In order to better understand children's experience with and usage of even, we conducted two corpus studies, in which we compare the features of our stimuli to tokens of even found in child and adult corpora. Our investigation resulted in three relevant findings:

\footnotetext{
${ }^{22}$ Attempts at modeling this contrast with a multinomial mixed-effects model unfortunately failed to converge.

${ }^{23}$ Our scoring of justifications was charitable toward adult-like interpretations or true guesses so as to minimize the chance of inflating the rate of opposite responses. ${ }^{24}$ Recall that the rate of opposite responses in Experiment 1 was inflated due to the ambiguity of the filling stories. Reanalysis of the results from Experiment 1 that accounts for this inflation in fact demonstrates a similar polarity asymmetry: opposite responses are primarily concentrated in negative environments ( 3 in POS vs. 17 in NEG).
} 
1. Children produce even essentially adult-like (error-free) as early as 3 years of age, hence much earlier than they comprehend even in an adult-like manner.

2. Adults show a use-bias for even in negative environments (here, "use-bias" refers to a bias toward using a word in a particular environment). Children, by contrast, initially favor even in positive environments and acquire the adult use-bias for negative even in the ages of 4-5.

3. The form of stimuli in our comprehension study instantiate a low-frequency use pattern for even in child and child-directed speech.

To study the distribution of even in both child-produced speech and child-directed speech ${ }^{25}$, we examined token instances of even in the American English CHILDES corpus (MacWhinney, $2000)^{26}$. Data were analyzed using childes-coder (Sanchez et al., 2019; Gowda, 2020), which presents instances of target words along with their broader contexts, and provides an interface for users to save metadata about these instances in a database (Supplementary Figure 6). Instances of even produced by speakers (marked Target_Child for child-produced speech, and marked Mother, Father, Adult, Uncle, Grandmother, Aunt, Grandfather, Family_Friend, or Teacher for child-directed speech) were coded by mutual agreement among the authors for several criteria, including:

1. Presence of negation: $\{$ Yes, No, Unclear $\}$

2. Order of negation and even: $\{\mathrm{N} / \mathrm{A}$, even-NEG, NEG-even, Unclear\}

3. Whether negation is sentential: $\{$ Yes, No, Unclear $\}$

4. Order of even and the subject: \{even-Subj, Subj-even, Unclear\}

5. The focus associate of even: \{Subject, Object, Verb, Adjunct, Unclear\}

6. The likelihood inference: $\{$ Most-Likely, Least-Likely, Unclear\}

In addition to examining the surrounding context of each instance of even to determine the correct coding, we also made use of the metadata and audio recordings available in CHILDES.

\subsection{Results}

Our comprehension studies focused on the interaction between polarity and the likelihood inference in even sentences. Because we coded tokens of even in production by both polarity and the contextually salient likelihood inference, we can similarly investigate this interaction in production. Results for childproduced speech are summarized in the first four rows of Table 9. Results for child-directed adult speech are in the last row.

In Table 9, for both adults and children, the rates of "opposite" uses of even-most-likely inferences for even in positive environments and least-likely inferences in negative environments-are so low that they are essentially absent from our findings. This is quite striking given that we see robust rates of

\footnotetext{
${ }^{25}$ Here, we use "child-directed speech" to refer to all utterances produced by non-child speakers in the CHILDES corpora. Thus, this data includes all speech in CHILDES that a child was exposed to, not necessarily just speech that was specifically directed at a child.

${ }^{26}$ Due to metadata consistency issues, data from the MacWhinney corpus was excluded from the analysis of both child-produced and child-directed speech.
}

TABLE 9 | Child and child-directed (adult) production of even in negative and positive environments between 3 and 6 years old.

\begin{tabular}{|c|c|c|c|c|c|}
\hline Age group & $\begin{array}{l}\text { Sentence } \\
\text { polarity }\end{array}$ & Inference: Least-likely & Most-likely & Unclear & Total \\
\hline \multirow{3}{*}{3} & POS & 47 & 1 & 3 & 51 \\
\hline & NEG & 1 & 17 & & 18 \\
\hline & POS & 99 & 1 & 2 & 104 \\
\hline \multirow[t]{3}{*}{4} & NEG & 1 & 161 & 5 & 167 \\
\hline & Unclear & 1 & 1 & 1 & 3 \\
\hline & POS & 25 & (1) & 1 & (29) 28 \\
\hline \multirow[t]{2}{*}{5} & $N E G$ & & 42 & & 42 \\
\hline & Unclear & 1 & 1 & & 2 \\
\hline \multirow{3}{*}{6} & POS & 6 & & 2 & 8 \\
\hline & NEG & & 23 & 1 & 24 \\
\hline & POS & 754 & (57) 0 & 31 & (842) 785 \\
\hline \multirow[t]{2}{*}{ Adults } & $N E G$ & 16 & 1,143 & 27 & 1,186 \\
\hline & Unclear & 1 & 2 & 7 & 10 \\
\hline
\end{tabular}

Figures in gray show numbers before post-hoc reanalysis (cf. footnote 27).

TABLE 10 | The ratio of negative to positive even sentences by age.

\begin{tabular}{lccc}
\hline Age group & NEG:Pos even ratio & NEG count & Pos count \\
\hline 3 & 0.35 & 18 & 51 \\
4 & 1.6 & 167 & 104 \\
5 & 1.5 & 42 & 28 \\
6 & 3 & 24 & 8 \\
Adult & $(1.5) 1.6$ & $(1,186) 1,243$ & 785
\end{tabular}

In adults, we include downward-entailing sentences which lack sentential negation in the NEG figures, with ratios/counts excluding these sentences in gray.

opposite responses in comprehension and suggests that opposite responses are, in fact, a comprehension phenomenon ${ }^{27}$.

Another salient pattern we observe in both child and childdirected corpus data is the overall prevalence of negative-even sentences (Table 10). Children start off using even in positive environments more often than in negative environments at age 3 . According to a chi-squared test for homogeneity, the distribution of positive and negative uses of even in 3yos is significantly different from adults $(p<0.001)$, while there is no significant difference $(p>0.1)$ between the distribution of positive and negative even sentences in 4,5 , and 6yos and adults. That is, by age 4 , children appear to have an adult-like use-bias for even in negative environments.

Lastly, we investigated several features of our comprehension study stimuli in the corpus and found that our stimuli instantiate

\footnotetext{
${ }^{27}$ In actuality, because our coding schema kept track of the polarity of the environment rather than upward/downward entailment, there were higher numbers of most-likely inferences in apparent positive environments, as indicated in Table 9 by the gray figures. However, these are not true opposite responses because most-likely inferences are predicted by the grammar in downward entailing environments more generally, whether or not there is sentential negation. Indeed, a study of most-likely responses in positive sentences for adults shows that they all involve downward entailing environments.
} 
a low-frequency usage pattern for even. Our stimuli can be described according to the following feature specification:

1. Focus associate $=$ subject

2. Even precedes the subject

3. Even precedes negation

We parametrically compared our stimuli to other even constructions by coding for whether a given utterance with even focused the subject, whether it contained sentential negation, and the linear order of even, the focus associate, and negation (if applicable). Supplementary Table 1 in the Supplementary Material shows that subject focus was relatively infrequent compared to VP or object focus. When the subject was focused, however, pre-subject even was preferred to post-subject even.

In addition to the placement of the subject with respect to even, we can compare the placement of even with respect to negation (Supplementary Table 2). Even follows negation in the majority of cases. When this requirement interacts with the previous tendency for subject-associating even to appear presubject, we see a preference for maintaining both constraints, resulting in not even. Examples with both subject focus and sentential negation were therefore most often of the form in (16b). Our stimuli, by contrast, took the form in $(16 a)^{28}$.

(16) a. Even Linda $F$ didn't write to me. (dispreferred)

b. Not even Linda $F$ wrote to me. (preferred)

\subsection{Analysis and Discussion}

In our corpus studies, children as young as 3yo produced even as if they were adults ${ }^{29}$. Taking their behavior at face value, and assuming Snyder (2007)'s principle of Grammatical Conservatism, this should not be possible unless they have identified a grammatical basis for even's scalar inferences.

(17) Grammatical Conservatism: Children do not begin making productive use of a new grammatical construction in their spontaneous speech until they have both determined that the construction is permitted in the adult grammar, and identified the adult's grammatical basis for it. (Snyder, 2007)

The fact that they exhibit non-adult-like behavior in comprehension should therefore not indicate that they lack a grammar entirely, as is argued by Kim (2011), Ito (2012) e.g. Indeed we argue that children must have a grammar of sorts for even, which happens to invite at times non-adult-like behavior in comprehension. Moreover, children must have some appreciation for which cells in the space of inferences allowed by their grammar are not available to adults, or else they would not be so adult-like in production. We will explore this in section 6 .

\footnotetext{
${ }^{28}$ Supplementary Table 1 in the Supplementary Material contains all tokens of even in which any type of negation was present; sentential or constituent. Even-NEG order was mostly available for clauses with constituent rather than sentential negation.

${ }^{29}$ Although in this paper we only present CHILDES data from ages 3-6, adult-like production of even is apparent as early as 2 years old, with 32 instances of Pos even sentences and 16 instances of NEG even sentences.
}

This conclusion is supported by another finding from our corpus studies, namely the fact that our comprehension task stimuli instantiate a low-frequency use pattern for even in both child and adult speech. Children's lack of experience with our even sentences, however, apparently did not deter them. Children ages 4-6 still offered adult-like responses as the dominant response pattern in our comprehension studies. We must therefore conclude that their command of even is quite sophisticated. They are able to abstract away from the particular even sentences that they hear and use most frequently, and generalize to other less-frequent uses.

Lastly, our corpus studies offered a perspective on the polarity asymmetry observed in our comprehension studies. Adults apparently produce even approximately 1.5 times more often in negative environments than positive environments, which translates into a comparative abundance of negative even in children's input. We propose that children show (slightly) better rates of comprehension in negative environments because they have more experience trying to interpret negative even. This effect is, however, less pronounced than our other findings because of the natural tension between inherent knowledge and experience. The proposed child grammar of even affords children the same abstract knowledge of even in negative as well as positive environments, which accounts for their overall adultlike competence. Their performance with even in real time, however, can be marginally affected by their confidence with each polarity environment ${ }^{30}$.

\section{LEARNING SCALAR INFERENCES}

The previous sections presented novel findings from a series of comprehension and corpus studies which significantly enrich our knowledge of the empirical landscape of how even is acquired. The picture that emerges reveals a surprisingly intricate developmental path.

Our evidence from the comprehension studies suggests that children as young as 4 years of age systematically draw scalar inferences that are comparable in nature to those generated by the adult grammar of even. Interestingly, their knowledge of the environmental conditions controlling when to draw which type of inference (least-likely-to in positive environments and most-likely-to/least-likely-to-not in negative environments) is not completely adult-like at this age. This gives rise to a comprehension behavior that runs at times directly opposite to that of adult speakers.

With regard to the basic effect of polarity on the nature of the scalar inference, we saw a rather striking lack of opposite scalar inferences in the corpus data. Even occurrences of even produced by 3 yo conform to the adult pattern. This is quite surprising since we know from the comprehension experiments that opposite

\footnotetext{
${ }^{30}$ Recall that this effect is fragile in our experiments (i.e., statistically present in Experiment 1 but only present as a trend in Experiment 2). This fragility might be due to a rather fine-grained effect of experience. It is conceivable that the absence of the ability modal and the presence of the present perfect make our stimuli in Experiment 2 even less well-represented in the input than the stimuli in Experiment 1. If this is true, it would thereby delay the advantage of negative environments to a later age when enough of such cases have been encountered.
} 
inferences are allowed by their developing grammar as late as age 6. Furthermore, children ages 4-6 were adult-like in production to the extent that they showed an adult-like use-bias for negative even, indicating that they are also sensitive to the conversational settings in which even sentences are predominantly used.

To explain why the acquisition of even unfolds along such an intricate path is a non-trivial task. It involves specifying the initial hypothesis space that the learners start out with as well as the final state that characterizes the adult grammar of even. Furthermore, learners must identify the relevant evidence as well as develop or adopt strategies that together enable them to transform the former cognitive structure into the latter. Though we are not yet in a position to offer a full account of the acquisition of even that lives up to all of these demands, we do think that our findings allow us to make significant progress toward that goal.

\subsection{Initial Hypothesis Space for Even}

The findings from our comprehension studies provide persuasive information about the nature of the initial hypothesis space: it needs to allow for all four combinations of likelihood inferences and polarity of the environment to be expressible by even, Table 1A. A simple way of implementing such a grammar would be to postulate a polysemous even which can freely occur in positive and negative environments.

Our argument in support of this conclusion is straightforward. We found both adult-like and non-adult-like scalar inferences in the earliest stages of comprehending even in both positive and negative environments. In other words, we saw that all four cells in Table 1A are utilized as soon as learners start to appreciate the scalar nature of even. Importantly, we saw that all four inference patterns occurred stably throughout an extended period of learning, and we found them to be regularly accompanied by reasoned justifications that referenced the relevant scale properties. This shows that the non-adult-like responses (just like the adult-like responses) were sanctioned by the developing grammar. Thus, they should be analyzed as exemplars that are predicted by the initial hypothesis space rather than as errors whose source is unrelated to the grammar of the learner.

\subsection{Adult Grammar of Even}

From the perspective of the two competing views on the adult grammar described in section 2, the initial hypothesis space in Table 1A can be described as an as yet unconstrained form of the grammar predicted by the ambiguity theory. Recall that the main tenet of the ambiguity theory is that even can in principle carry a least-likely as well as a most-likely inference. The distribution of these variants needs to be constrained via the addition of a grammatical feature (in this case an NPI-feature on most-likely even). Without that addition, the distribution of even would remain unconstrained allowing for all logically possible combinations to be realized, Table $\mathbf{1 A}$.

An as yet unconstrained version of the grammar predicted by the scope theory, by contrast, is not a viable option for the initial hypothesis space since it makes only three of the four required cells available, Table 1B. The reason is, again, transparent. The main tenet of the scope theory is that even can only carry a leastlikely inference. In combination with negation we can generate a most-likely inference if even out-scopes negation since the resulting least-likely-to-not inference is equivalent to a mostlikely-to inference. However, without the presence of negation, the scope theory can only generate a least-likely inference, which leaves the most-likely inferences in positive environments unaccounted for.

Though we have identified (on empirical grounds) a greater similarity between the initial hypothesis space for even and the ambiguity theory, it would be unjustified to conclude at this point that the ambiguity theory has to be correct for the adult grammar of even. Both theories of adult even are, in fact, compatible with the initial state postulated for children. They simply require different transformations on the initial state. Deciding which one offers the better account for the adult grammar, therefore, depends on what the actual steps are that allow learners to transform the initial hypothesis space (Table 1A) into either the constrained version of Table $\mathbf{1 A}$ or Table $\mathbf{1 B}$.

To arrive at an ambiguity grammar of adult even, the learner must replace the polysemous even with two separate evens, each specified for a particular likelihood inference and level of polarity sensitivity (NPI or unmarked). Under the scope theory, learning amounts to eliminating the possibility of even triggering a most-likely inference altogether. This is arguably a simpler transformation on the initial hypothesis space. However, it yields the target grammar only at the expense of adopting an unprecedented constraint on the syntactic scope of even.

Importantly, both theories also have to explain why other logically possible combinations of likelihood inferences and polarity-sensitivity/scope constraint are never selected by learners of English even. Below we argue that a plausible source to rule out unattested combinations is the limited conversational utility of those combinations. Interestingly, these considerations will also provide us with a possible account of why acquiring the adult grammar takes relatively long and what might be responsible for the puzzling productioncomprehension asymmetry we have observed.

\subsection{Pragmatics of Likelihood-Inferences}

Throughout the paper we have described the scalar inferences triggered by even in terms of likelihoods- "least-likely-to" in positive environments and "most-likely-to/least-likely-to-not" in negative environments. In doing so, we adopted the terminology of Karttunen and Peters (1979) which is intuitive and sufficiently transparent to characterize the differences between the various scalar inferences we have encountered. Whether likelihood is (always) the correct way to characterize the dimension of the scalar inferences of even is, however, debated in the literature. Alternatives include various formulations of expectedness, noteworthiness, informativity as well as scales introduced by gradable predicates ${ }^{31}$. We cannot provide a full assessment of

\footnotetext{
${ }^{31}$ See Fillmore, 1965; Fauconnier, 1975; Kay, 1990; Giannakidou, 2007; Rullmann, 2007; Greenberg, 2016, among others. Refinements of, or alternatives to likelihoodbased characterization can be envisioned that are consistent with our findings. Choosing among them is, however, not topical for us here.
} 
this debate here. Instead, our aim is to clarify the connection between the inferred relative likelihood of a proposition and its noteworthiness in a given conversational situation. This will be sufficient to diagnose the conversational status of the adult-like as well as the opposite scalar inferences we have observed.

Taking a closer look at the opposite inferences preschoolers draw in our comprehension studies, we observe that they are not simply absent from the adult grammar of even, but are in fact conversationally odd if we try to render their content anyway. Compare, for example, (18), which features the content of the adult-like scalar inferences via an appositive relative clause, to its rather odd sounding counterpart in (19), which features the "opposite" content of even's inferences.

\section{(18) Adult-like inferences}

a. Everybody has reached the book, including Jessiepillar, who was the least likely to have done so.

b. Nobody has reached the book, including Jessiepillar, who was the most likely to have done so.

(19) Opposite inferences

a. \# Everybody has reached the book, including Jessiepillar, who was the most likely to have done so.

b. "Nobody has reached the book, including Jessiepillar, who was the least likely to have done so.

We propose that a pragmatic explanation of this contrast can provide insight into the factors that help learners constrain their initial hypothesis space for even. To see how, let us examine the conversational context in which our even sentences were uttered.

Recall that the three characters in our stories either all succeeded at the relevant task or they all failed. This fact was highlighted explicitly with a universal statement immediately preceding the even sentence. The truth-conditional content of the even sentence was therefore redundant, which puts the burden to provide conversational utility for the utterance squarely on its not-at-issue content. A question we might ask is whether a pragmatic requirement on conversational utility constrains the space of possible likelihood inferences at all.

Given the oddity of (19), we argue that it does. Moreover we propose that this oddity is derived if a connection between a character's likelihood of success with some notion of propositional noteworthiness is important to adult-like competence with even. To see why, it is important to consider the context in which the even sentence occurs very carefully.

After the universal statement but before the even sentence is uttered, the context contains a proposition of the form, Every $x$ in $C$ is such that $x$ has reached the book. If the modal horizon against which the likelihood inference were evaluated contained all and only the verifying situations characterized by the universal statement, the likelihood inference would be moot. Comparing the relative likelihood of Jessiepillar has reached the book to Some other $x$ in $C$ has reached the book is almost non-sensical because both sentences are already true in the context.

What allows the likelihood inference to be meaningful and useful is to consider a context in which it was not given that any character would reach a book. In other words, for the even sentence to have a sensible inference, it must take as its context variable a set of propositions that does not contain the universal statement that preceded the even sentence in our experiment. But what drives this move? Why does a listener bother to accommodate a different common ground in which to make sense of the likelihood inference, rather than just ignore it? We propose that this move is connected to a notion of propositional noteworthiness.

We propose that when an adult listener hears an even sentence in our context, they detect its redundancy and ask, what makes this proposition worth repeating? I.e., what makes this result surprising or deserving of comment? It is this question that allows the listener to specify a useful common ground in which to consider the likelihood inference. The logic goes as follows: in order for "Jessiepillar has reached the book" to be noteworthy, it must be unexpected. Jessiepillar must therefore be the character whose success was least likely.

Notice, however, that there is no way to connect to a mostlikely inference on this logic, hence capturing the oddness of (19). Considering characters who were likely to succeed in no way explains why emphasizing those characters' success is interesting.

If this is the type of pragmatic reasoning that adults employ generally in a conversation, opposite responses are predicted to be infelicitous, thus providing insight into why the grammar of even is constrained to just two adult-like inferences. Therefore, the content that our child comprehenders end up with when they select the opposite character is odd from the perspective of the adult grammar.

To clarify, for Jessiepillar to be singled out even though her height doesn't justify it, as happens when children provide opposite responses, is of course logically possible ${ }^{32}$. However, for adult speakers this requires a different "backstory," e.g., Jessiepillar might be the most/least motivated of the three to do what it takes to reach the book making her the most/least likely to succeed/fail despite her height. Our stories did not provide any useful information about the characters other than their height, however. Thus, adult comprehenders are stuck with Jessiepillar's height as the only available basis for anchoring the likelihood inference triggered by even. They therefore pick the shortest character to be Jessiepillar when all candidates succeed and the tallest when all of them fail.

For our child comprehenders the situation is different. While they may be practiced enough conversationalists to consider a common ground in which a likelihood inference is meaningful, their grammar overgenerates. Because their grammar allows for a least-likely as well as a most-likely specification of the scalar inference regardless of the polarity of the sentence, they consider both the tallest and the shortest character as grammatically viable candidates for Jessiepillar. This is only possible, however, if we assume that children are less attuned to the relevant conversational pragmatics than adults are, thus allowing both options to remain viable in our conversational setting. Thus, they can in principle choose the character at either end of the scale.

\footnotetext{
${ }^{32}$ Our cases are not characterized by entailment relations between alternatives. In such cases, opposite responses would only be possible at pains of accepting a contradiction. See e.g., (Lahiri, 1998).
} 
With regard to why children are less adept at this kind of pragmatic reasoning than adults, a number of options seem plausible. For instance, it may be that "explicit" Theory of Mind level reasoning about the motivations of speakers, which is required to detect this sort of pragmatic oddness, is not yet fully developed or sufficiently practiced ${ }^{33}$. Alternatively, or additionally, it may be that children's processing resources for this kind of reasoning are still not up to full capacity. Whatever the true underlying causes, it seems reasonable to characterize their pragmatic reasoning as more tolerant than those of adults toward not knowing exactly what the speaker had in mind when they issued their even sentence ${ }^{34}$. A willingness to proceed in a state of partial ignorance leaves both inferences in play making both extrema live possibilities. Of course, this follows only if the developing grammar generates both types of inferences in both environments to begin with. On our proposal this is so because their grammar allows for all four cells of the initial hypothesis space to be expressible by even ${ }^{35}$.

The fact that opposite choices occur at all is therefore predicted on the basis of children's more limited conversational experience compared to adults. However, the fact that opposite choices occur less frequently than adult-like choices may be seen as a reflection of those choices being less optimal even from the perspective of the learner. After all, these opposite choices require a willingness to proceed without having figured out exactly what the speaker meant with their even sentence ${ }^{36}$.

Turning to the question of how children actually acquire the adult grammar of even, the following picture emerges: learning, under the present view, is a function of becoming more adept at recognizing conversational goals and more intolerant when those goals are not identified during comprehension. In other words, the more pragmatically skilled a learner is, the better they will be at recognizing and recording the specific conversational setting in which even sentences are used. This growing conversational confidence favors the adult grammar, which does not support opposite inferences, and correspondingly discriminates against a

\footnotetext{
${ }^{33}$ Though it is now widely accepted that some aspects of Theory of Mind reasoning, often called "implicit" Theory of Mind inferences, are in place earlier than our age range (cf. Onishi and Baillargeon, 2005) it is plausible that the relevant skills in our task include assessing a speaker's conversational assumptions and goals, which has been argued to come online much later in development and hence are likely to scale with the mount of practice young reasoners have, see e.g., (Perner and Roessler, 2012).

${ }^{34}$ See Katsos and Bishop, 2011 for a similar notion that children are pragmatically more tolerant than adults.

${ }^{35} \mathrm{We}$ have evidence from the adult control studies that the adult grammar, by contrast, does not provide access to all four cells. Rather, the adult error pattern we have observed is compatible with only three of the four cells in the space (Table 1B). This potentially indicates that the adult grammar is most like the scope theory, since only one type of error-least-likely-to inferences in negative environments-occurred. Most-likely-to inferences in positive environments never occurred, which is straightforwardly predicted by the scope theory. The ambiguity theory, by contrast, rules them out by a mechanism-licensing of NPI-that is known to be error prone during processing (Drenhaus et al., 2005).

${ }^{36}$ If we assume that whenever they proceed in a state of ignorance they are guessing which character they should pick our observed rate of opposite responses of $25 \%$ translates into a rate $50 \%$ of not being able to figure out what exactly the speaker had in mind. To assess whether this is a reasonable estimate would, however, require a more fleshed out theory of what makes pragmatic reasoning of this sort difficult for our learner.
}

grammar that does support opposite inferences. Eventually, the absence of evidence in favor of opposite inferences is taken by the learner to suggest an adjustment to the grammar to ensure that sentences that would generate opposite inferences are no longer generated to begin with ${ }^{37}$.

Turning to the question from section 6.2, i.e., why learners do not consider other logically possible adult grammars with different combinations of likelihood inferences and polarity, two factors emerge: (1) no data from the input supports such grammars, and (2) the learner's own pragmatic knowledge discourages them.

Last but not least, the present perspective also allows us to sketch a plausible account of the production-comprehension asymmetry. Recall that we never see children use even in a non-adult way, even at the earliest stages. Specifically, they underutilize the opposite inferences that their grammar apparently licenses.

A key difference between production and comprehension is that the speaker knows what that intended message is, while the comprehender has to figure out what the message is that the speaker intended ${ }^{38}$. Our account allows us to exploit this difference directly: we proposed that opposite inferences surface during comprehension when the listener is unable to figure out what the speaker's conversational goals might be (e.g., why they singled out Jessiepillar), but is nevertheless willing to go along with the task at hand (in our case selecting one of the three characters $)^{39,40}$. Importantly, we did not require that children not appreciate the connection between likelihood and expectedness. Indeed, the fact they mostly interpret our target sentences in an adult way suggests that they do, just not as reliably as adults. This means that we can reasonably assume that when they issue an even sentence they do so with the intent to convey information that rides on the intuitive connection between likelihood and expectedness.

\footnotetext{
${ }^{37}$ Our proposal is part of the growing literature on the role of pragmatics in language acquisition which has uncovered a great deal of pragmatic sophistication that young learners bring to the task of language acquisition in variety of different situations, ranging from referential word learning (e.g., Horowitz and Frank, 2015; Sullivan and Barner, 2015; Sullivan et al., 2019) to speech act pragmatics and its role in the acquisition of propositional attitudes (Hacquard and Lidz, 2018). What the precise relation of our proposal is to the type of pragmatic reasoning in these cases is not immediately obvious since expunging a grammatical option that is underutilized because speakers tend to not highlight likely outcomes bears little resemblance to determining when and how a novel word is used by a speaker to refer to a novel object or the cases Hacquard and Lidz (2018)'s "pragmatic syntactic bootstrapping hypothesis" is meant to account for.

${ }^{38}$ See especially (Hendriks, 2014) for discussion of production-comprehension asymmetries in language acquisition.

${ }^{39}$ See Aravind, 2018 for evidence that children in our age range who are otherwise quite astute at picking up the status of presupposed information as contextually entailed nevertheless prefer a fully redundant reading of an utterance over one that is informative only at pains of accommodation.

${ }^{40}$ The error pattern we observed in our adult comprehenders-opposite responses in negative environments-can be understood in a similar vein if we assume, as seems plausible, that our web-based task environment invites shallow processing as a form of satisficing, (Ferreira, 2003). Shallow processing will generate scalar inferences for even but may not require a full reconstruction of the (imagined) speaker's conversational purpose in singling out Jessiepillar.
} 


\section{CONCLUSION}

This paper has advanced a view of the scalar inferences associated with even in child grammar that stands in contrast with much prior literature. Previous acquisition studies of even and scalar inferences treated children's non-adult-like behavior as evidence of simple confusion about even and likelihood inferences in discourse. Our studies present evidence to the contrary. Children are not simply confused. They are, in fact, rather keenly sensitive to the scalar nature of even and generate robustly both least-likely and most-likely inferences very early on. They are non-adult-like only in that they exhibit more tolerance to uses of even during comprehension where the speaker's conversational goals carried by even are left unresolved.

On our view, there is nothing difficult per se about detecting scalar inferences. As soon as children learn to associate them with the particle even, they immediately access a relevant hypothesis space of scalar inferences associated with even along the lines of (Rooth, 1985)'s ambiguity theory (Table 1A). This is evident from their "error" patterns in our comprehension studies, as well as the absence of such errors in production.

We presented two comprehension studies that shared Kim (2011)'s "Guess who?" format. In these experiments, children as young as 4yo performed well above chance. Most notably, children in our age range predominantly chose either the mostor the least-likely character to succeed in any given story, but rarely chose the middle character. They additionally justified their choices with comments that demonstrated a sensitivity to scalar properties about the characters. This behavior is predicted by the space of inferences in Table $\mathbf{1 A}$, but is not expected if children are merely guessing.

Further motivation for this treatment of child non-adult-like behavior comes from our adult control studies. Adults likewise were occasionally susceptible to "opposite" likelihood inferences, and justified these choices with normal reference to the scalar properties of the characters. We argued that this wouldn't be possible unless these inferences were made in principle available by the grammar.

In addition to the comprehension experiments, we conducted two corpus studies that examined tokens of even in child and child-directed speech. Strikingly, neither adults nor children exhibited "opposite of adult-like" uses of even. We argue on

\section{REFERENCES}

Aravind, A. (2018). Presuppositions in context (Ph.D. thesis). Cambridge, MA: MIT.

Crnič, L. (2009). Getting even (Ph.D. thesis). Cambridge, MA: MIT.

Crnič, L. (2014). Non-monotonicity in NPI licensing. Nat. Lang. Semant. 22, 169-217. doi: 10.1007/s11050-014-9104-6

Drenhaus, H., Frisch, S., and Saddy, D. (2005). "Processing negative polarity items: when negation comes through the backdoor," in Linguistic Evidence, eds S. Kepser and M. Reis (Berlin: Mouton de Gruyter), 145-164. doi: 10.1515/9783110197549.145

Drummond, A. (2012). Ibex: A Web Interface for Psycholinguistic Experiments 6. Available online at: https://github.com/addrummond/ibex (accessed April 9, 2018). the basis of this production-comprehension asymmetry that children not only hypothesize a space of inferences like that in Table 1A, but they also command some of the knowledge necessary to constrain this space (or else they wouldn't be so adult-like in production).

Learning to transform the space in Table 1A to the adult grammar amounts to making use of that knowledge in comprehension as well as production. This is a gradual process of becoming increasingly intolerant to certain inferences, as they become increasingly confident in their ability to reason about, and identify, speakers' conversational goals.

\section{DATA AVAILABILITY STATEMENT}

The datasets presented in this study can be found in online repositories. The names of the repository/repositories and accession number(s) can be found https:/github.com/ MITLanguageAcquisitionLab/even.

\section{ETHICS STATEMENT}

The studies involving human participants were reviewed and approved by MIT COUHES. Written informed consent to participate in this study was provided by the participants' legal guardian/next of kin.

\section{AUTHOR CONTRIBUTIONS}

EN was responsible for initial conception of research question. EN, YG, and LR were involved in data collection. EN, YG, LR, and $\mathrm{MH}$ were involved in design of experimental material. EN, YG, and $\mathrm{MH}$ were involved in theory development. YG was involved in statistical analysis. EN, YG, and $\mathrm{MH}$ were involved in writing. $\mathrm{MH}$ is the PI of the hosting lab. All authors contributed to the article and approved the submitted version.

\section{SUPPLEMENTARY MATERIAL}

The Supplementary Material for this article can be found online at: https://www.frontiersin.org/articles/10.3389/fcomm. 2020.593634/full\#supplementary-material

Fauconnier, G. (1975). Pragmatic scales and logical structure. Linguist. Inq. 6, 353-375.

Ferreira, F. (2003). The misinterpretation of noncanonical sentences. Cogn. Psychol. 47, 164-203. doi: 10.1016/S0010-0285(03) 00005-7

Fillmore, C. J. (1965). Entailment Rules in Semantic Theory. POLA report, Ohio State University Research Foundation.

Francis, N. (2018). "Presupposition-denying uses of even," in Proceedings of SALT 28, eds S. Maspong, B. Stefánsdóttir, K. Blake, and F. Davis (Ithaca, NY: CLC Publications), 161-176. doi: 10.3765/salt.v28i0.4409

Gast, V., and van der Auwera, J. (2011). Scalar additive operators in the languages of Europe. Language 87, 2-54. doi: 10.1353/lan.2011.0008

Gelman, A., and Rubin, D. B. (1992). Inference from iterative simulation using multiple sequences. Stat. Sci. 7, 457-472. doi: 10.1214/ss/1177011136 
Giannakidou, A. (2007). The landscape of even. Nat. Lang. Linguist. Theory 25, 39-81. doi: 10.1007/s11049-006-9006-5

Gowda, Y. (2020). Childes-Coder: Utility for Coding Data From CHILDES Corpora. Available online at: https://github.com/tlonic/childes-coder (accessed March 13, 2020),

Greenberg, Y. (2015). "Even, comparative likelihood and gradability," in Amsterdam Colloquium, eds T. Brochhagen, F. Roelofsen, and N. Theiler (Amsterdam), 147-156.

Greenberg, Y. (2016). A novel problem for the likelihood-based semantics of even. Semant. Pragmat/ 9, 1-28. doi: 10.3765/sp.9.2

Greenberg, Y. (2018). A revised gradability semantics for even. Nat. Lang. Semant. 26, 51-83. doi: 10.1007/s11050-017-9140-0

Guerzoni, E. (2004). Even-NPIs in yes/no questions. Nat. Lang. Semant. 12, 319-343. doi: 10.1007/s11050-004-8739-0

Hacquard, V., and Lidz, J. (2018). Children's attitude problems: bootstrapping verb meaning from syntax and pragmatics. Mind Lang. 34, 73-96. doi: $10.1111 /$ mila. 12192

Hadfield, J. D. (2010). MCMC methods for multi-response generalized linear mixed models: the MCMCglmm R package. J. Stat. Softw. 33, 1-22. doi: $10.18637 /$ jss.v033.i02

Halle, M., and Marantz, A. (1993). "Distributed morphology and the pieces of inflection," in The View from Building 20: Essays in Linguistics in Honor of Sylvain Bromberger, eds K. L. Hale, S. J. Keyser, and S. Bromberger (Cambridge, MA: MIT Press), 111-176.

Hendriks, P. (2014). Asymmetries between Language Production and Comprehension, volume 42 of Studies in Theoretical Psycholinguistics. Cambridge, MA: Springer. doi: 10.1007/978-94-007-6901-4

Herburger, E. (2000). What Counts: Focus and Quantification. Cambridge, MA: MIT Press. doi: 10.7551/mitpress/7201.001.0001

Horn, L. (1969). A Presuppositional Analysis of Only and Even. Chicago, IL: Chicago Linguistic Society.

Horn, L. (1972). On the semantic properties of logical operators in English (Ph.D. thesis). University of California, Los Angeles, CA, United States.

Horn, L. (1989). A Natural History of Negation. Chicago, IL: University of Chicago Press.

Horowitz, A. C., and Frank, M. C. (2015). Young children's developing sensitivity to discourse continuity as a cue for inferring reference. J. Exp. Child Psychol. 129, 84-97. doi: 10.1016/j.jecp.2014.08.003

Ito, M. (2012). Japanese-speaking children's interpretation of sentences containing the focus particle datte 'even': conventional implicatures, QUD, and processing limitations. Linguistics 50, 105-151. doi: 10.1515/ling-2012-0004

Karttunen, L., and Peters, S. (1979). "Conventional implicature," in Syntax and Semantics, eds C. K. Oh and D. A. Dineen (New York, NY: Academic Press), $1-56$.

Katsos, N., and Bishop, D. V. (2011). Pragmatic tolerance: implications for the acquisition of informativeness and implicature. Cognition 120, 67-81. doi: 10.1016/j.cognition.2011.02.015

Kay, P. (1990). Even. Linguist. Philos. 13, 59-111. doi: 10.1007/BF00630517

Kim, S. (2011). Focus particles at syntactic, semantic and pragmatic interfaces: the acquisition of only and even in English (Ph.D. thesis). Honolulu, HI: University of Hawaii.

Krifka, M. (1991). "A compositional semantics for multiple focus constructions," in Proceedings of SALT I, eds S. K. Moore and A. Z. Wyner (Ithaca, NY: CLC Publications), 127-158. doi: 10.3765/salt.v1i0.2492

Kruschke, J. K., Aguinis, H., and Joo, H. (2012). The time has come: Bayesian methods for data analysis in the organizational sciences. Organ. Res. Methods 15, 722-752. doi: 10.1177/1094428112457829
Lahiri, U. (1998). Focus and negative polarity in Hindi. Nat. Lang. Semant. 6, 57-123. doi: 10.1023/A:1008211808250

MacWhinney, B. (2000). The CHILDES Project: Tools for Analyzing Talk. Transcription Format and Programs, Vol. 1. Mahwah, NJ: Lawrence Erlbaum. doi: $10.1162 /$ coli.2000.26.4.657

Nicenboim, B., and Vasishth, S. (2016). Statistical methods for linguistic research: foundational ideas-part II. Lang. Linguist. Compass 10, 591-613. doi: 10.1111/lnc3.12207

Onishi, K. H., and Baillargeon, R. (2005). Do 15-month-old infants understand false beliefs? Science 308, 255-258. doi: 10.1126/science. 1107621

Perner, J., and Roessler, J. (2012). From infants to children's appreciation of belief. Trends Cogn. Sci. 16, 519-525. doi: 10.1016/j.tics.2012.08.004

Plummer, M., Best, N., Cowles, K., and Vines, K. (2006). CODA: convergence diagnosis and output analysis for MCMC. R News 6, 7-11.

Rooth, M. (1985). Association with focus (Ph.D. thesis). University of Massachusetts, Amherst, MA, United States.

Rooth, M. (1996). "Focus," in The Handbook of Contemporary Semantic Theory, ed S. Lappin (Oxford: Blackwell Publishers), 271-297. doi: 10.1111/b.9780631207498.1997.00013.x

Rullmann, H. (1997). "Even, polarity, and scope," in Papers in Experimental and Theoretical Linguistics, eds M. Gibson, G. Wiebe, and G. Libben (Edmonton, AB: University of Alberta), 40-64.

Rullmann, H. (2007). What does even even mean? (Ms). University of British Columbia, Vancouver, BC, Canada.

Sanchez, A., Meylan, S. C., Braginsky, M., MacDonald, K. E., Yurovsky, D., and Frank, M. C. (2019). Childes-DB: a flexible and reproducible interface to the child language data exchange system. Behav. Res. Methods 51, 1928-1941. doi: 10.3758/s13428-018-1176-7

Schwarz, B. (2005). Scalar additive particles in negative contexts. Nat. Lang. Semant. 13, 125-168. doi: 10.1007/s11050-004-2441-0

Snyder, W. (2007). Child Language: The Parametric Approach. Oxford: Oxford University Press.

Sullivan, J., and Barner, D. (2015). Discourse bootstrapping: preschoolers use linguistic discourse to learn new words. Dev. Sci. 19, 63-75. doi: 10.1111/desc.12289

Sullivan, J., Boucher, J., Kiefer, R. J., Williams, K., and Barner, D. (2019). Discourse coherence as a cue to reference in word learning: evidence for discourse bootstrapping. Cogn. Sci. 43:e12702. doi: 10.1111/cogs. 12702

von Stechow, A. (1991). "Current issues in the theory of focus," in Semantics: An International Handbook of Contemporary Research, eds A. von Stechow and D. Wunderlich (Berlin: Walter deGruyter), 804-825.

Wilkinson, K. (1996). The scope of even. Nat. Lang. Semant. 4, 193-215. doi: $10.1007 / \mathrm{BF} 00372819$

Conflict of Interest: The authors declare that the research was conducted in the absence of any commercial or financial relationships that could be construed as a potential conflict of interest.

Copyright (c) 2020 Gowda, Newman, Rosenstein and Hackl. This is an open-access article distributed under the terms of the Creative Commons Attribution License (CC $B Y)$. The use, distribution or reproduction in other forums is permitted, provided the original author(s) and the copyright owner(s) are credited and that the original publication in this journal is cited, in accordance with accepted academic practice. No use, distribution or reproduction is permitted which does not comply with these terms. 